\title{
Analyse de la dégradation thermique du Poly(éther imide)
}

\author{
Emilie Courvoisier ${ }^{1,2}$, Yoann Bicaba ${ }^{1}$, and Xavier Colin ${ }^{2, *}$ \\ 1 SAFRAN Composites, 33 avenue de la Gare, 97601 Itteville, France \\ 2 Laboratoire PIMM, Arts et Métiers ParisTech, 151 boulevard de l'Hôpital, 75013 Paris, France
}

Reçu le 7 juillet 2017 / Accepté le 9 janvier 2018

\begin{abstract}
Résumé. La dégradation thermique du PEI a été étudiée dans de larges intervalles de température (entre 180 et $250{ }^{\circ} \mathrm{C}$ ) et de pression partielle d'oxygène (entre 0,21 et 50 bars). Tout d'abord, les mécanismes de vieillissement thermique ont été analysés et élucidés par spectroscopie IRTF et par calorimétrie différentielle (DSC) sur des films de PEI suffisamment minces (entre 10 et $60 \mu \mathrm{m}$ d'épaisseur) pour s'affranchir totalement des effets de la diffusion d'oxygène. Comme attendu, et par analogie avec d'autres polymères aromatiques de structure chimique similaire, l'oxydation se produit préférentiellement sur les groupes méthyle de l'unité isopropylidène du motif bisphenol A, causant la disparition de leur bande d'absorption IR caractéristique à $2970 \mathrm{~cm}^{-1} \mathrm{et} \mathrm{la}$ croissance d'une nouvelle bande d'absorption IR centrée à $3350 \mathrm{~cm}^{-1}$ et attribuée aux groupes alcool. De plus, l'oxydation conduit successivement à une prédominance relative des coupures de chaîne (diminution de $T_{g}$ ) et de la réticulation (augmentation de $\mathrm{T}_{\mathrm{g}}$ ). Enfin, les conséquences de l'oxydation sur les propriétés élastiques ont été analysées et élucidées par micro-indentation sur des sections droites préalablement polies de plaquettes de PEI de $3 \mathrm{~mm}$ d'épaisseur. Cependant, l'augmentation du module d'Young dans la couche superficielle oxydée est principalement due à un vieillissement physique.
\end{abstract}

Mots clés : PEI / thermo-oxydation / coupures de chaîne / réticulation / module d'Young

\begin{abstract}
Analytical study of the thermal degradation of poly(ether imide). The thermal degradation of PEI has been studied in wide ranges of temperature (between 180 and $250^{\circ} \mathrm{C}$ ) and oxygen partial pressure (between 0.21 and 50 bars). First of all, the thermal ageing mechanisms have been analysed and elucidated by FTIR spectroscopy and by differential scanning calorimetry (DSC) on sufficiently thin PEI films (between 10 and $60 \mu \mathrm{m}$ thickness) to be totally free of the effects of oxygen diffusion. As expected, and by analogy with other aromatic polymers of similar chemical structure, oxidation occurs preferentially on the methyl groups of the isopropylidene unit of the bisphenol A part, leading to the disappearance of their characteristic IR absorption band at $2970 \mathrm{~cm}^{-1}$ and the growth of a new IR absorption band centered at $3350 \mathrm{~cm}^{-1}$ and attributed to alcohol groups. In addition, oxidation leads successively to a relative predominance of chain scissions (decrease in $\mathrm{T}_{\mathrm{g}}$ ) and crosslinking (increase in $\mathrm{T}_{\mathrm{g}}$ ). Finally, the consequences of oxidation on the elastic properties have been analysed and elucidated by micro-indentation on preliminarily polished cross-sections of PEI plates of $3 \mathrm{~mm}$ thickness. However, the increase in Young's modulus in the superficial oxidized layer is mainly due to a physical ageing.
\end{abstract}

Keywords: PEI / thermal oxidation / chain scissions / crosslinking / Young's modulus

\section{Introduction}

La compétition sur le marché aéronautique dépend d'enjeux à la fois économiques et écologiques tels que la réduction des coûts, de la masse embarquée et de la consommation de carburant. C'est pourquoi, depuis son origine, l'industrie aéronautique est à la recherche de nouveaux matériaux plus légers aux propriétés thermomécaniques élevées. Dans ce contexte, l'emploi de

\footnotetext{
* e-mail: xavier.colin@ensam.eu
}

matériaux composites constitués de matrice organique renforcée par des fibres de verre ou de carbone (CMO) n'a cessé de progresser dans le but de remplacer peu à peu les matériaux métalliques. D'abord conçus pour la fabrication de pièces de structure (ailes, queue, fuselage, etc.), les CMO sont maintenant envisagés pour des applications dans des environnements thermochimiques de plus en plus sévères, comme les zones proches des sources de chaleur (environnements de moteur d'avion). Cependant, ils ne pourront être utilisés que si leur durabilité à long terme est clairement démontrée. L'utilisation de matrices thermoplastiques présente plusieurs avantages, comme l'intégration de 
fonction ou encore l'assemblage de pièces de même nature ou de natures différentes. Parmi les matrices thermoplastiques pouvant répondre au cahier des charges, le poly(éther imide) (PEI) apparaît comme un sérieux candidat. En effet, il s'agit d'un polymère amorphe de structure hautement aromatique lui confèrant à la fois de hautes performances thermomécaniques $\left(\mathrm{T}_{\mathrm{g}} \approx 214^{\circ} \mathrm{C}\right)$ et une grande résistance chimique à la plupart des solvants usuels (acétone, toluène, acétate d'éthyle, etc.) et aux fluides aéronautiques (en particulier, le Skydrol). La dégradation thermique du PEI a été peu étudiée dans la littérature, quasi-exclusivement à l'état caoutchoutique au-dessus de $300^{\circ} \mathrm{C}$ sous atmosphère inerte ou faiblement oxygénée (typiquement dans l'air).

L'oxydation du PEI, comme des polymères aromatiques de structure chimique similaire, se produit principalement au niveau des groupes méthyle de l'unité isopropylidène $\mathrm{du}$ motif bisphénol $\mathrm{A}$, qui comporte les atomes d'H les plus labiles [1,2]. L'oxydation conduit à la formation d'une grande variété de produits de dégradation qui sont détectés par spectroscopie IRTF. Il s'agit des carbonyles, notamment des cétones aromatiques (à $\left.1690 \mathrm{~cm}^{-1}\right)$ et aliphatiques $\left(1724 \mathrm{~cm}^{-1}\right)$ et des anhydrides $\left(1840\right.$ et $\left.1860 \mathrm{~cm}^{-1}\right)$, ainsi que d'hydroxyles (3553 et $3514 \mathrm{~cm}^{-1}$ ) [2-6]. La disparition des bandes d'absorption IR caractéristiques de l'unité isopropylidène (liaison $\mathrm{C}$ $\mathrm{CH}_{3}$ à $1186 \mathrm{~cm}^{-1}$ ) et des groupes méthyle (liaison $\mathrm{C}-\mathrm{H}$ à 2870,2933 et $2970 \mathrm{~cm}^{-1}$ ) a aussi été observée [3,5].

Des mécanismes de coupure de chaîne ont été proposés pour expliquer la formation de la grande variété de produits volatils. Les coupures se produisent au niveau des liaisons $\mathrm{C}-\mathrm{C}$ de l'unité isopropylidène, mais aussi au niveau des liaisons éther entre les cycles phényle et phthalimide [1,69]. Elles prédominent largement sur la réticulation en début d'exposition, mais deviennent finalement négligeables devant la réticulation à plus long terme [1,5,7,10,11]. La recombinaison bimoléculaire des radicaux méthylène serait la principale source de réticulation.

Cet article présente une analyse à la fois multi-échelles et multi-techniques de la dégradation thermique du PEI à des températures plus basses et des pressions partielles d'oxygène plus fortes que celles étudiées jusqu'à présent dans la littérature. Une attention particulière est portée aux conséquences de la dégradation sur deux propriétés thermomécaniques clés en aéronautique : la température de transition vitreuse et le module d'Young.

\section{Matériaux et techniques}

Le matériau d'étude est le PEI Ultem 1010 fourni par la société Sabic sous forme de granulés. Des plaques de $3 \mathrm{~mm}$ d'épaisseur ont été injectées au Pôle Européen de Plasturgie (PEP) d'Oyonnax à partir de ces granulés. Les plaques ont été ensuite découpées au microtome LEICA RM2255 pour obtenir des films suffisamment minces (typiquement entre 10 et $60 \mu \mathrm{m}$ d'épaisseur) garantissant une oxydation homogène dans toute l'épaisseur. La cinétique d'oxydation du PEI a été étudiée à l'état caoutchoutique à 220 et $250^{\circ} \mathrm{C}$, mais aussi à l'état vitreux à 180 et $200^{\circ} \mathrm{C}$, sous des pressions partielles d'oxygène comprises entre 0,21 bar (dans des étuves à ventilation d'air forcée, régulées à $\pm 1^{\circ} \mathrm{C}$ ) et 50 bars (dans des autoclaves). Tous les films ont été périodiquement sortis des enceintes de vieillissement et refroidis à température ambiante dans un dessiccateur contenant du silica-gel pour éviter une reprise en humidité avant d'être caractérisés. À noter que des échantillons plus épais (plaquettes de $3 \mathrm{~mm}$ d'épaisseur) ont aussi été exposés à 220 et $250^{\circ} \mathrm{C}$ dans l'air dans des étuves ventilées pour mettre en évidence les profils d'oxydation. Dans tous les cas, les conséquences de l'oxydation ont été analysées par plusieurs techniques complémentaires depuis l'échelle moléculaire jusqu'à l'échelle macroscopique, en passant par les échelles macromoléculaire et microscopique.

Les modifications de la structure moléculaire ont été suivies par spectroscopie IRTF en mode transmission. Les spectres IRTF des films de PEI ont été mesurés avant et après vieillissement afin de pouvoir détecter et quantifier la disparition de groupes chimiques initialement présents dans la matrice PEI et la formation des principaux produits d'oxydation. Les analyses ont été réalisées à l'aide d'un appareil Frontier de chez Perkin Elmer dans un domaine spectral s'étendant de 400 à $4000 \mathrm{~cm}^{-1}$, après avoir moyenné les 16 enregistrements obtenus avec une résolution minimale de $4 \mathrm{~cm}^{-1}$. Le veillissement thermique du PEI conduit à deux principales modifications du spectre IRTF (Fig. 1). Tout d'abord, l'intensité des bandes d'absorption IR à 2970, 2935 et $2875 \mathrm{~cm}^{-1}$, qui sont caractéristiques des liaisons $\mathrm{C}-\mathrm{H}$ des groupes $-\mathrm{CH}_{3}$ de l'unité isopropylidène initialement présents dans la matrice PEI, mais aussi des groupes $-\mathrm{CH}_{2}-$ formés au cours du vieillissement, diminue. La seconde modification est l'apparition et la croissance d'une large bande d'absorption IR centrée à $3350 \mathrm{~cm}^{-1}$ et attribuée à la liaison $\mathrm{O}-\mathrm{H}$ des groupes alcool.

Rappelons que la concentration $\mathrm{C}$ des différents produits de dégradation est liée à l'absorbance Abs de leur bande IR par la loi de Beer-Lambert:

$$
C=\frac{A b s}{e \times \varepsilon}
$$

où e est l'épaisseur de l'échantillon (en cm) et $\varepsilon$ le coefficient d'extinction molaire dont les valeurs moyennes sont accessibles dans la littérature: $\varepsilon(\mathrm{O}-\mathrm{H}$ des alcools $)=90 \mathrm{~L} \cdot \mathrm{mol}^{-1}$. $\mathrm{cm}^{-1}[12,13]$ et $\varepsilon\left(\mathrm{C}-\mathrm{H}\right.$ de $\left.\mathrm{CH}_{3}\right)=42 \mathrm{~L} \cdot \mathrm{mol}^{-1} . \mathrm{cm}^{-1}[14]$.

Les conséquences de l'oxydation sur l'architecture macromoléculaire ont été suivies par analyse calorimétrique (DSC). Les thermogrammes DSC des films de PEI ont été mesurés avant et après vieillissement afin d'accéder à l'évolution de la température de transition vitreuse $\left(\mathrm{T}_{\mathrm{g}}\right)$. Les analyses ont été effectuées à l'aide d'un calorimètre Q1000 de chez TA Instruments entre 25 et $300^{\circ} \mathrm{C}$ avec une vitesse de chauffe de $10^{\circ} \mathrm{C} \cdot \mathrm{min}^{-1}$ sous flux d'azote. La valeur de $\mathrm{T}_{\mathrm{g}}$ a été prise au point d'inflexion de la courbe de variation du flux de chaleur avec la température.

Enfin, l'impact du vieillissement sur les propriétés élastiques (principalement sur le module d'Young) a été déterminé par des essais de micro-indentation sur les sections droites préalablement polies des plaquettes de $3 \mathrm{~mm}$ d'épaisseur. Les plaquettes ont été découpées suivant leur épaisseur et enrobées à froid dans une résine acrylique commerciale KM-V, qui a ensuite été réticulée pendant $12 \mathrm{~h}$ 

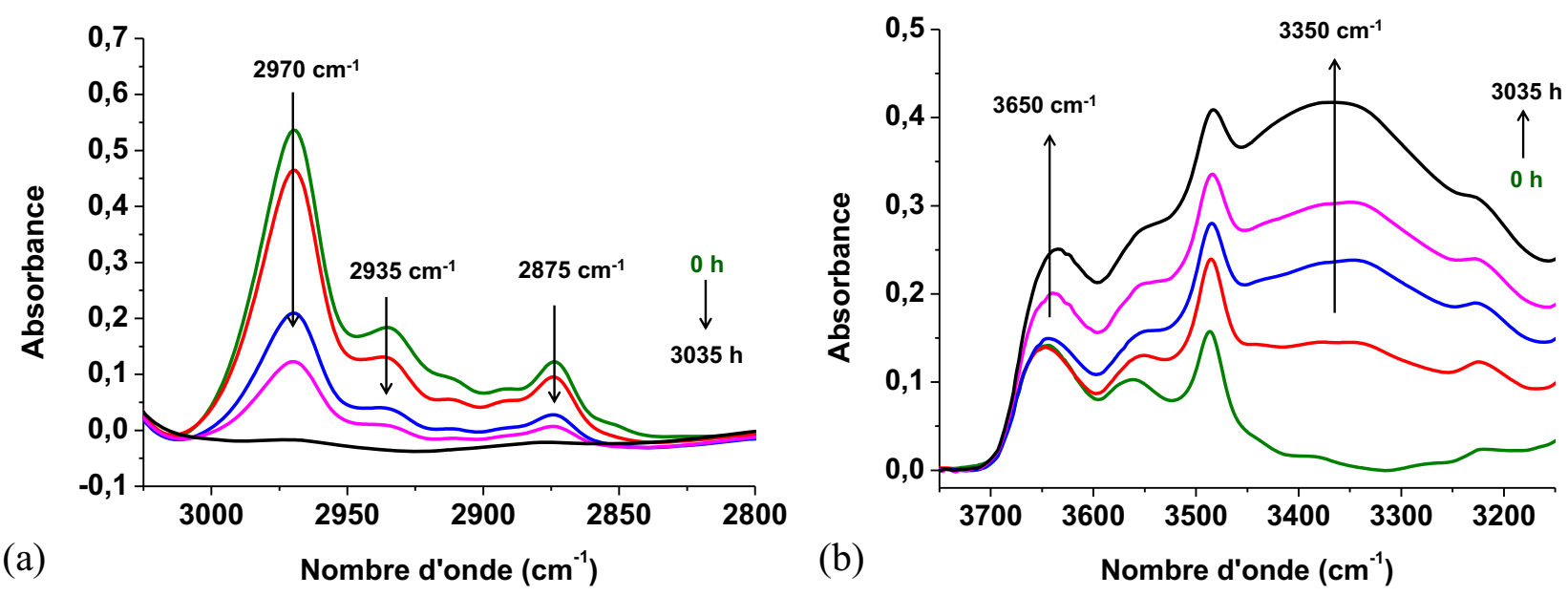

Fig. 1. Modification des régions des liaisons $\mathrm{C}-\mathrm{H}$ (a) et $\mathrm{O}-\mathrm{H}$ (b) du spectre IRTF du PEI au cours de son vieillissement thermique dans l'air à $250^{\circ} \mathrm{C}$.

Fig 1. Changes in the regions of $C-H(a)$ and $O-H$ bonds (b) of the FTIR spectrum of PEI during its thermal ageing in air at $250^{\circ} C$.

sous vide primaire à température ambiante. Les sections droites ont été polies à l'aide d'une polisseuse MECAPOL P320 en utilisant tout d'abord, une série de papiers abrasifs en particules de carbure de silicium de taille décroissante (typiquement de granulométrie 80 à 2400). Enfin, une finition miroir a été obtenue à l'aide de pâtes de particules de diamant de taille décroissante (typiquement de 3 à $0,25 \mu \mathrm{m})$. Les indentations ont ensuite été réalisées à l'aide du Micro Indentation Tester (MHT) de chez CSM Instrument équipé d'une pointe en diamant de type Vickers de géométrie pyramidale avec une force de $500 \mathrm{mN}$, et des vitesses de charge et décharge de $1000 \mu \mathrm{m} \cdot \mathrm{min}^{-1}$. Une pause de 5 secondes a été systématiquement appliquée entre la charge et la décharge. Le logiciel d'exploitation Indentation 4.37 donne directement accès à la valeur du module réduit du matériau $\left(\mathrm{E}_{\mathrm{r}}\right)$ qui est calculée selon la méthode d'Oliver \& Pharr [3,15-17]:

$$
E_{r}=\frac{\sqrt{\pi} S}{2 \beta \sqrt{A_{c}}},
$$

où $\mathrm{S}$ est la pente initiale de la courbe de décharge, $\beta$ est un facteur de forme dépendant du type d'indenteur $(\beta=1,012$ dans le cas d'un indenteur de type Vickers) et $A_{c}$ est l'aire de contact projetée. Cette dernière grandeur est directement donnée par le logiciel d'exploitation. Elle dépend de la profondeur de contact de l'indenteur sur l'échantillon et de la géométrie de l'indenteur.

Le module élastique local a été déterminé à partir du module réduit $E_{r}$ et de la relation suivante:

$$
E=\frac{1}{\frac{1-v^{2}}{E_{r}}-\frac{1-v_{i} 2}{E_{i}}},
$$

où $v$ est le coefficient de Poisson du PEI vierge égal à 0,44 (donnée fournisseur), et $\nu_{\mathrm{i}}$ et $\mathrm{E}_{\mathrm{i}}$ sont le coefficient de Poisson et le module d'Young de l'indenteur en diamant, respectivement égaux à 0,07 et $1141 \mathrm{GPa}$.
Le module élastique global $E_{\text {Echant }}$ de l'échantillon a ensuite été déduit des $\mathrm{N}$ valeurs locales $\mathrm{E}(\mathrm{j})$ composant le profil de micro-indentation à l'aide de la relation suivante :

$$
\mathrm{E}_{\mathrm{Echant}}=\frac{1}{N} \sum_{j=1}^{N} E(j) .
$$

Dans cette étude, $\mathrm{E}_{\text {Echant }}$ a été utilisé comme module d'Young de l'échantillon après avoir vérifié, par traction uniaxiale, qu'il donne une très bonne estimation de ce dernier. En effet, il a été trouvé que leurs valeurs respectives ne diffèrent que d'un facteur 0,7 .

\section{Résultats et discussion}

\subsection{Modifications de la structure moléculaire}

La figure 2 rapporte l'évolution des concentrations en alcools (à $3345 \mathrm{~cm}^{-1}$ ) et en liaisons C-H $\left(2970 \mathrm{~cm}^{-1}\right)$ au cours du vieillissement du PEI dans l'air entre 200 et $250^{\circ} \mathrm{C}$. Comme attendu, la température accélère la cinétique d'oxydation du PEI.

La consommation des liaisons C-H s'explique à la fois par l'élimination (sous forme de méthane) des groupes $\mathrm{CH}_{3}$ de l'unité isopropylidène par rupture thermolytique des liaisons $\mathrm{C}-\mathrm{CH}_{3}[1,6-8]$, mais aussi par la propagation préférentielle de l'oxydation sur les groupes $\mathrm{CH}_{3}$. En effet, rappelons que l'énergie de dissociation d'une liaison $\mathrm{C}-\mathrm{H}$ d'un groupe $\mathrm{CH}_{3}$ n'est que de $347 \mathrm{~kJ} . \mathrm{mol}^{-1}$ lorsqu'il est situé à proximité d'un cycle aromatique, contre $465 \mathrm{~kJ}$. $\mathrm{mol}^{-1}$ pour une liaison $\mathrm{C}-\mathrm{H}$ aromatique [18].

À titre d'exemple, la figure 3 compare l'évolution des concentrations en alcools et en liaisons $\mathrm{C}-\mathrm{H}$ dans l'air à $220^{\circ} \mathrm{C}$. On constate que les alcools apparaissent dès que les liaisons $\mathrm{C}-\mathrm{H}$ commencent à disparaître. De manière plus générale, les évolutions de ces deux espèces sont parfaitement corrélées: les vitesses (d'accumulation ou de disparition) deviennent maximales et se ralentissent 

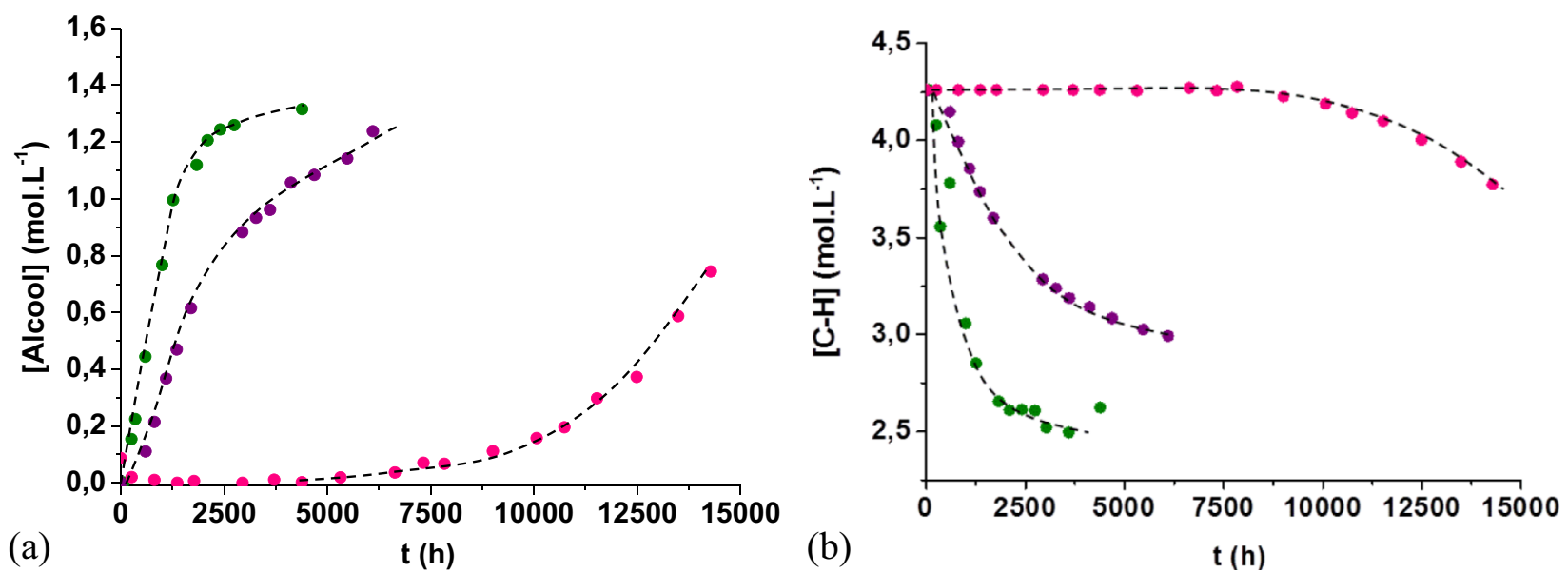

Fig. 2. Évolution des concentrations en alcools (a) et en liaisons C-H (b) au cours du vieillissement thermique du PEI dans l'air à 200 (en rose), 220 (violet) et $250^{\circ} \mathrm{C}$ (vert).

Fig 2. Changes in the concentrations of alcohols (a) and $C-H$ bonds (b) during the thermal ageing of PEI in air at 200 (in pink), 220 (violet) and $250^{\circ} \mathrm{C}$ (green).

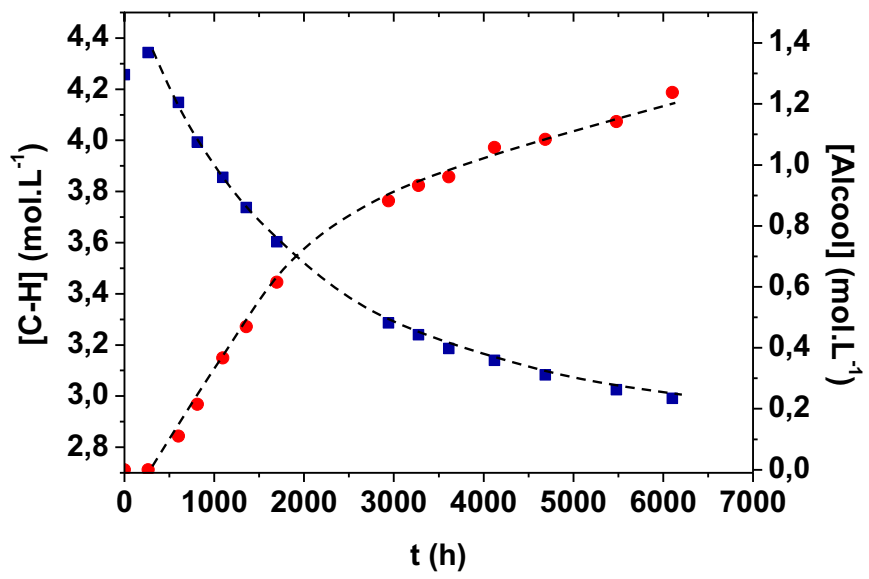

Fig. 3. Comparaison des évolutions de la concentration des liaisons $\mathrm{C}-\mathrm{H}(\mathbf{\square})$ et des alcools $(\mathbf{O})$ au cours du vieillissement thermique du PEI dans l'air à $220^{\circ} \mathrm{C}$.

Fig 3. Comparison between the changes in the concentration of $\mathrm{C}-\mathrm{H}$ bonds (-) and alcohols (O) during the thermal ageing of PEI in air at $220^{\circ} \mathrm{C}$.

brutalement, pour tendre vers une valeur quasi-constante, après les mêmes durées d'exposition. Ce résultat confirme que les alcools se forment par oxydation des groupes $\mathrm{CH}_{3}$ comme déjà décrit dans la littérature pour les polymères contenant le motif bisphénol A dans leur structure chimique, en particulier pour le PEI et le polycarbonate (PC) [1,4].

Deux indicateurs de vieillissement ont été déterminés graphiquement pour l'ensemble des courbes cinétiques. Il s'agit du temps d'induction $\left(t_{i}\right)$ marquant le début de la phase d'auto-accélération, et de la vitesse maximale d'oxydation $\left(\mathrm{r}_{\mathrm{ox}}\right)$ correspondant à la pente maximale des courbes cinétiques. Ces deux indicateurs ont été portés sur les graphes d'Arrhenius de la figure 4. Malgré la présence d'une transition physique séparant l'état vitreux de l'état caoutchoutique $\left(\mathrm{T}_{\mathrm{g}}=214^{\circ} \mathrm{C}\right)$ entre 200 et $250^{\circ} \mathrm{C}$, ils semblent tous les deux obéir à une loi d'Arrhenius.
Le tableau 1 récapitule les énergies d'activation de $t_{i}$ et $\mathrm{r}_{\mathrm{ox}}$ dans l'air entre 200 et $250^{\circ} \mathrm{C}$ déterminées pour les liaisons $\mathrm{C}-\mathrm{H}$ et les alcools. Bien que les évolutions des concentrations de ces deux espèces soient intimement liées (voir Fig. 3), leurs énergies d'activation ne sont pas pour autant identiques. En effet, il apparaît clairement que la disparition des liaisons $\mathrm{C}-\mathrm{H}$ est davantage favorisée à haute température que la formation des alcools.

Pour mettre en évidence l'influence de la pression partielle d'oxygène sur la cinétique d'oxydation du PEI, des analyses IRTF ont été réalisées sur des films vieillis sous des pressions partielles d'oxygène comprises entre 0,21 (dans l'air) et 50 bars entre 180 et $220^{\circ} \mathrm{C}$. À titre d'exemple, la figure 5 rapporte l'évolution des concentrations en alcools et en liaisons $\mathrm{C}-\mathrm{H}$ à $220^{\circ} \mathrm{C}$. Il apparaît clairement que la pression partielle d'oxygène accélère la cinétique d'oxydation du PEI.

Â titre d'exemple, les valeurs de $t_{\mathrm{i}}$ et $\mathrm{r}_{\mathrm{ox}}$ déterminées pour les liaisons $\mathrm{C}-\mathrm{H}$ et les alcools à $200^{\circ} \mathrm{C}$ ont été portées en fonction de la pression partielle d'oxygène sur la figure 6 . On constate qu'elles sont comparables pour ces deux espèces. De plus, les courbes présentent une allure hyperbolique et atteignent un plateau de saturation qui est caractéristique du régime en excès d'oxygène à forte pression partielle d'oxygène. En d'autres termes, $t_{i}$ et $r_{o x}$ deviennent indépendants de la pression partielle d'oxygène au-dessus d'une valeur critique, notée $\mathrm{P}_{\mathrm{c}}$.

Les valeurs de $P_{c}$, ainsi que les valeurs de $t_{i}$ et $r_{o x}$ en excès d'oxygène, déterminées entre 180 et $220^{\circ} \mathrm{C}$ sont récapitulées dans le tableau 2. On note que l'excès d'oxygène n'est pas complètement atteint dans l'air (c'est-à-dire pour une pression de 0,21 bar d'oxygène) à 200 et $220^{\circ} \mathrm{C}$. Cependant, il apparaît clairement que $\mathrm{P}_{\mathrm{c}}$ est une fonction décroissante de la température. En effet, à $180^{\circ} \mathrm{C}$, l'excès d'oxygène n'est atteint qu'à partir de 20 bars d'oxygène.

La figure 7 présente les graphes d'Arrhenius de $t_{i}$ et $r_{o x}$ en excès d'oxygène entre 180 et $220^{\circ} \mathrm{C}$. Les énergies d'activation, rapportées au tableau 3, sont très proches 

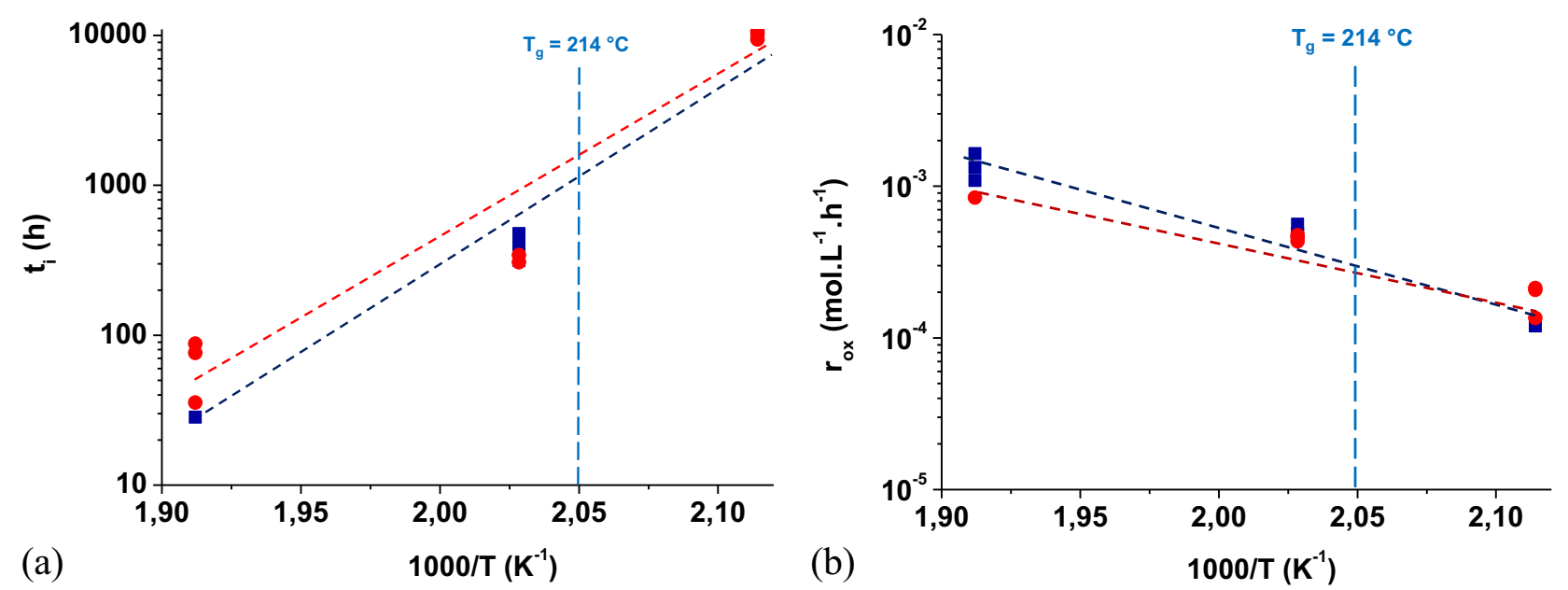

Fig. 4. Graphe d'Arrhenius des temps d'induction (a) et des vitesses maximales (b) de disparition des liaisons C-H ( $\mathbf{a})$ et d'accumulation des alcools (๑) dans l'air entre 200 et $250^{\circ} \mathrm{C}$.

Fig 4. Arrhenius diagram of the induction times (a) and the maximum rates (b) of the disappearance of $C$ - $H$ bonds (ロ) and accumulation of alcohols (O) in air between 200 and $250^{\circ} \mathrm{C}$.

Tableau 1. Énergie d'activation des temps d'induction $\left(\mathrm{t}_{\mathrm{i}}\right)$ et des vitesses maximales $\left(\mathrm{r}_{\mathrm{ox}}\right)$ de disparition des liaisons $\mathrm{C}-\mathrm{H}$ et d'accumulation des alcools dans l'air entre 200 et $250^{\circ} \mathrm{C}$.

Table 1. Activation energy of the induction times $\left(t_{i}\right)$ and the maximum rates $\left(r_{o x}\right)$ of the disappearance of $\mathrm{C}-\mathrm{H}$ bonds and accumulation of alcohols in air between 200 and $250^{\circ} \mathrm{C}$.

\begin{tabular}{lll}
\hline Groupements & $\begin{array}{l}\mathrm{E}_{\mathrm{a}} \mathrm{de} \mathrm{t}_{\mathrm{i}} \\
(\mathrm{kJ.mol}) \\
\text { dans l'air }\end{array}$ & $\begin{array}{l}\mathrm{E}_{\mathrm{a}} \mathrm{de} \mathrm{r}_{\mathrm{ox}} \\
\left(\mathrm{kJ.mol}^{-1}\right) \\
\text { dans l'air }\end{array}$ \\
\hline Liaisons C-H & 254 & 96 \\
Liaisons O-H (alcools) & 204 & 67 \\
\hline
\end{tabular}

pour les deux espèces considérées. Les écarts les plus importants observés dans l'air sont dûs à la variation en température de la pression critique. Ici aussi, la présence de la transition vitreuse $\left(\mathrm{T}_{\mathrm{g}}=214^{\circ} \mathrm{C}\right)$ entre 180 et $220^{\circ} \mathrm{C}$ ne semble pas altérer la validité de la loi d'Arrhenius.

L'ensemble de ces résultats expérimentaux milite en faveur du mécanisme de dégradation thermique à haute température proposé dans la littérature pour le PEI et le PC $[1,4,7,9]$, à savoir une oxydation amorcée à la fois par la rupture thermolytique des liaisons $\mathrm{C}-\mathrm{C}$ de l'unité isopropylidène et par la décomposition des hydroperoxydes, entraînant la disparition des groupes $\mathrm{CH}_{3}$ et la formation d'alcools. Rappelons que à haute température (typiquement au dessus de $200^{\circ} \mathrm{C}$ ), la décomposition des hydroperoxydes est principalement unimoléculaire [19]. La figure 8 résume ces deux voies de dégradation. Notons que l'oxydation pourrait également entraîner la formation de cétones par un rapide réarrangement (par coupure $\beta$ ) des radicaux alkoxyles $(\mathrm{PO} \bullet)$. Malheureusement, les cétones ne sont pas détectables par spectroscopie IRTF à cause de la présence initiale de deux larges bandes d'absorption IR entre 1680 et $1800 \mathrm{~cm}^{-1}$, attribuées aux vibrations d'élongation des liaisons $\mathrm{C}-\mathrm{O}$ des imides.

\subsection{Modifications de la structure macromoléculaire}

L'évolution des masses moléculaires pendant le vieillissement des polymères permet de mettre en évidence les modifications au niveau de l'architecture macromoléculaire, c'est-à-dire les coupures de chaîne (notées S) et les actes de réticulation (notés $\mathrm{X}$ ). Comme chaque coupure de chaîne fait apparaître une nouvelle chaîne tandis que chaque acte de réticulation fait disparaître une chaîne, on peut écrire :

$$
S-X=\frac{1}{M_{n}}-\frac{1}{M_{n 0}},
$$

où $\mathrm{M}_{\mathrm{n} 0}$ et $\mathrm{M}_{\mathrm{n}}$ sont respectivement les masses moléculaires moyennes en nombre avant et après vieillissement.

Cependant, il est impossible de suivre l'évolution des masses moléculaires du PEI pendant son vieillissement thermique par des méthodes d'analyse directes, de type chromatographique (CES ou GPC) ou viscosimétrique (en solution), car ce polymère réticule et devient donc rapidement insoluble dans les solvants usuels (en particulier, le dichlorométhane et le chloroforme). Nous avons donc eu recours à la calorimétrie différentielle (DSC) qui donne accès de manière indirecte à la valeur de $\mathrm{M}_{\mathrm{n}}$ connaissant la valeur de $\mathrm{T}_{\mathrm{g}}$ via la relation bien connue de Fox-Flory [20] :

$$
T_{g}-T_{g 0}=-k_{F F}\left(\frac{1}{M_{n}}-\frac{1}{M_{n 0}}\right),
$$

où $\mathrm{k}_{\mathrm{FF}}$ est la constante de Fox-Flory (exprimée en K.mol. $\mathrm{kg}^{-1}$ ) qui augmente avec la rigidité des chaînes. 

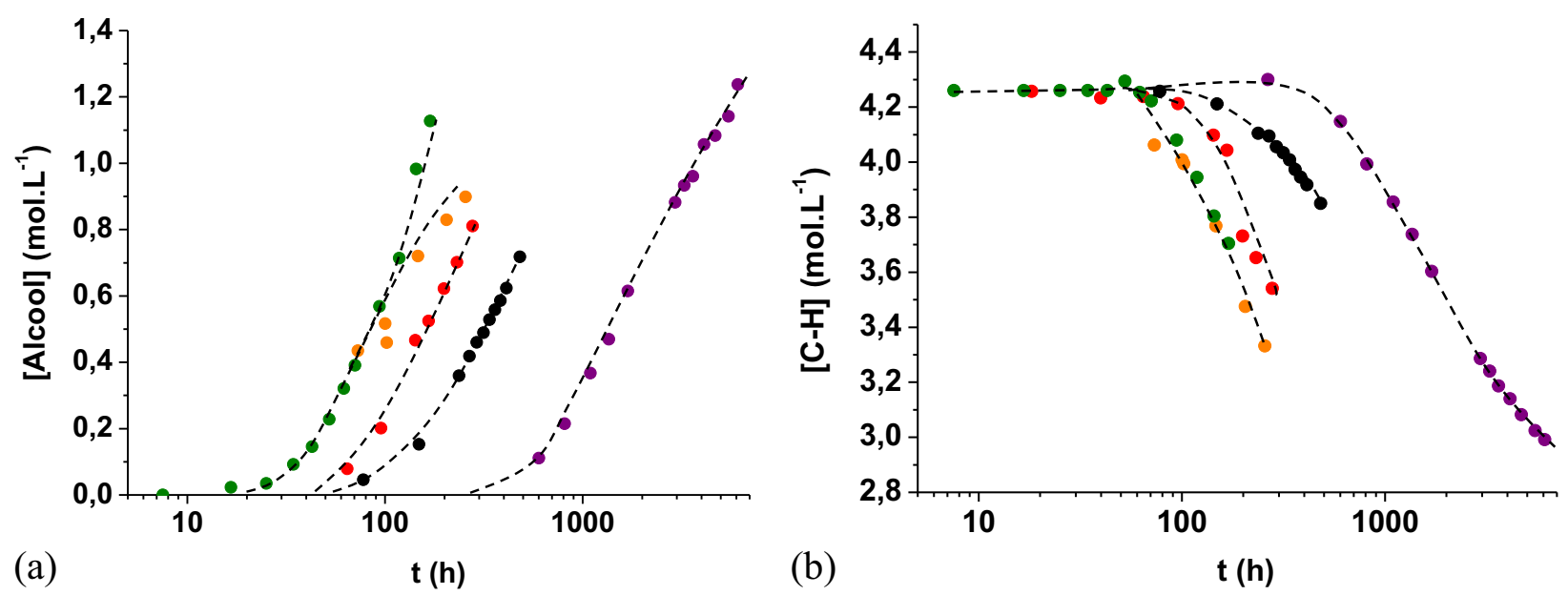

Fig. 5. Évolution des concentrations en alcools (a) et en liaisons $\mathrm{C}-\mathrm{H}$ (b) au cours du vieillissement thermique du PEI sous une pression de 0,21 bar (en violet), 0,4 bar (noir), 1 bar (rouge), 3 bars (orange) et 5 bars d'oxygène (vert) à $220^{\circ} \mathrm{C}$.

Fig 5. Changes in the concentrations of alcohols (a) and $C-H$ bonds (b) during the thermal ageing of PEI under an oxygen pressure of 0.21 bar (in violet), 0.4 bar (black), 1 bar (red), 3 bars (orange) and 5 bars (green) at $220^{\circ} \mathrm{C}$.
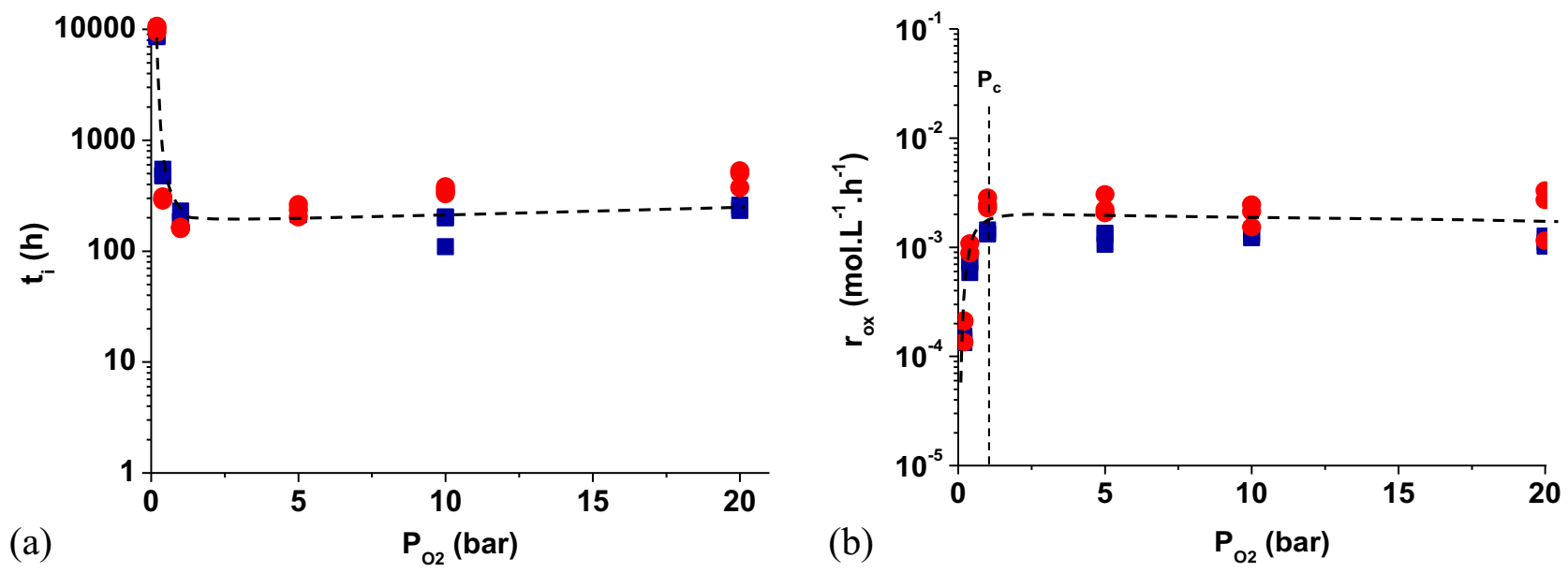

Fig. 6. Variation des temps d'induction (a) et des vitesses maximales (b) de disparition des liaisons C-H (ם) et d'accumulation des alcools (O) avec la pression partielle d'oxygène à $200^{\circ} \mathrm{C}$.

Fig 6. Changes in the induction times (a) and the maximum rates (b) of the disappearance of $C-H$ bonds ( $\mathbf{\square})$ and accumulation of alcohols (O) with the oxygen partial pressure at $200^{\circ} \mathrm{C}$.

Tableau 2. Pression critique $\left(\mathrm{P}_{\mathrm{c}}\right)$, temps d'induction $\left(\mathrm{t}_{\mathrm{i}}\right)$ et vitesse maximale $\left(\mathrm{r}_{\mathrm{ox}}\right)$ de disparition des liaisons $\mathrm{C}-\mathrm{H}$ ou d'accumulation des alcools en excès d'oxygène à 180, 200 et $220^{\circ} \mathrm{C}$.

Table 2. Critical pressure $\left(P_{C}\right)$, induction time $\left(t_{i}\right)$ and maximum rate $\left(r_{o x}\right)$ of the disappearance of $C-H$ bonds or accumulation of alcohols in oxygen excess at 180, 200 and $220^{\circ} \mathrm{C}$.

\begin{tabular}{llcl}
\hline $\begin{array}{l}\text { Température } \\
\left({ }^{\circ} \mathrm{C}\right)\end{array}$ & $\mathrm{P}_{\mathrm{c}}($ bar $)$ & $\begin{array}{l}\mathrm{t}_{\mathrm{i}} \text { en excès } \\
\mathrm{d}^{\prime} \mathrm{O}_{2}(\mathrm{~h})\end{array}$ & $\begin{array}{l}\mathrm{r}_{\mathrm{ox}} \text { en excès d'O } \\
\left(\mathrm{mol}_{2} \mathrm{~L}^{-1} \cdot \mathrm{h}^{-1}\right)\end{array}$ \\
\hline 180 & $\leq 20$ & 1519 & $0,59 \cdot 10^{-3}$ \\
200 & 1 & 257 & $1,8 \cdot 10^{-3}$ \\
220 & 0,6 & 53 & $4,3 \cdot 10^{-3}$ \\
\hline
\end{tabular}

$\mathrm{k}_{\mathrm{FF}}$ a été déterminé en moyennant les valeurs obtenues par trois méthodes différentes : la relation de Bicerano [21], la théorie du volume libre [22,23] et la théorie des copolymères [23]. Elle vaut: $\mathrm{k}_{\mathrm{FF}}=295 \pm 85 \mathrm{~K} . \mathrm{kg} \cdot \mathrm{mol}^{-1}$.

En combinant les équations 5 et 6 , on obtient la relation suivante entre $\mathrm{T}_{\mathrm{g}}$ et les nombres d'actes de coupure de chaîne et de réticulation :

$$
T_{g}-T_{g 0}=k_{F F}(X-S) .
$$

On voit que $\mathrm{T}_{\mathrm{g}}$ diminue avec le nombre de coupures de chaîne, mais augmente avec le nombre d'actes de réticulation.

La figure 9 rapporte l'évolution des écarts entre la $\mathrm{T}_{\mathrm{g}}$ et sa valeur initiale $\left(\mathrm{T}_{\mathrm{g}}-\mathrm{T}_{\mathrm{g} 0}\right)$ et entre les nombres d'actes de réticulation et de coupure de chaîne $(\mathrm{X}-\mathrm{S})$ pour les films de 

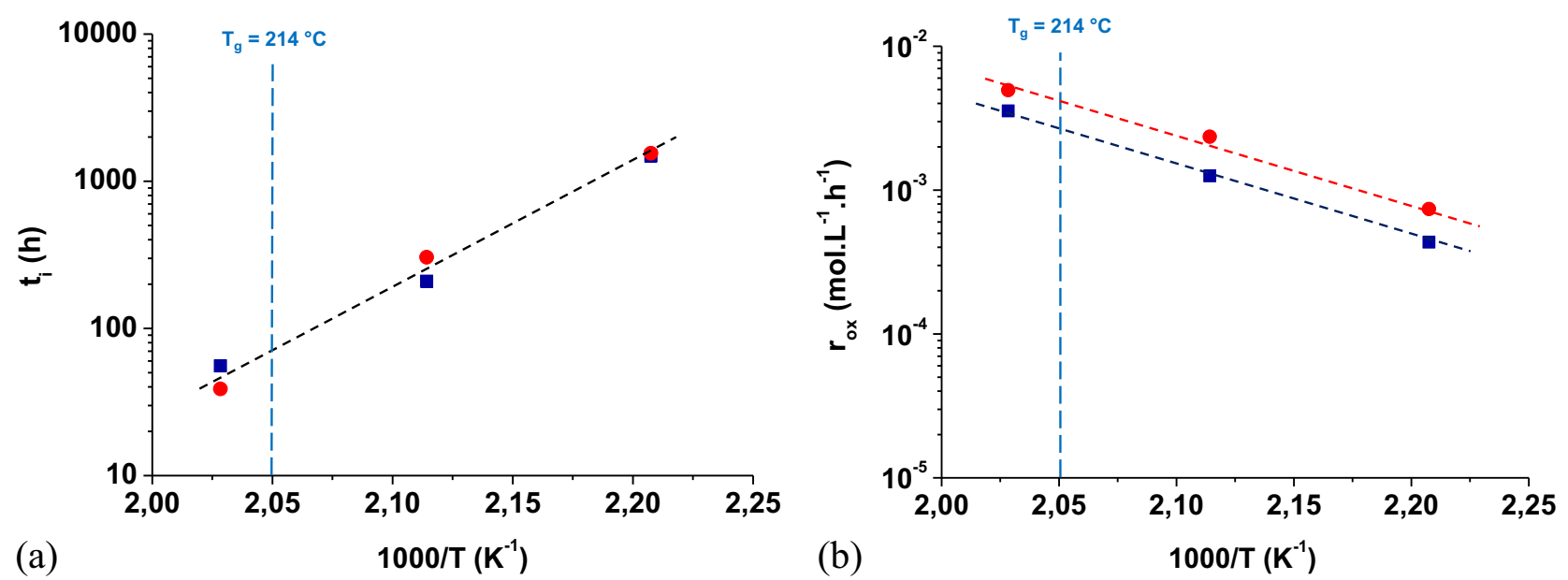

Fig. 7. Graphe d'Arrhenius des temps d'induction (a) et des vitesses maximales (b) de disparition des liaisons C-H ( $\mathbf{\square})$ et de formation des alcools $(\mathbf{O})$ en excès d'oxygène entre 180 et $220^{\circ} \mathrm{C}$.

Fig 7. Arrhenius diagram of the induction times (a) and the maximum rates (b) of the disappearance of $C-H$ bonds ( $\mathbf{\square})$ and formation of alcohols (O) in oxygen excess between 180 and $220^{\circ} \mathrm{C}$.

Tableau 3. Énergie d'activation des temps d'induction $\left(\mathrm{t}_{\mathrm{i}}\right)$ et des vitesses maximales $\left(\mathrm{r}_{\mathrm{ox}}\right)$ de disparition des liaisons $\mathrm{C}-\mathrm{H}$ et de formation des alcools en excès d'oxygène entre 180 et $220^{\circ} \mathrm{C}$.

Table 3. Activation energy of the induction times $\left(t_{i}\right)$ and the maximum rates $\left(r_{\text {ox }}\right)$ of the disappearance of $\mathrm{C}-\mathrm{H}$ bonds and formation of alcohols in oxygen excess between 180 and $220^{\circ} \mathrm{C}$

\begin{tabular}{lll}
\hline Groupements & $\begin{array}{l}\mathrm{E}_{\mathrm{a}} \text { de t }_{\mathrm{i}} \\
\left(\mathrm{kJ} \cdot \mathrm{mol}^{-1}\right) \\
\text { en excès d'O }\end{array}$ & $\begin{array}{l}\mathrm{E}_{\mathrm{a}} \text { de r }_{\mathrm{ox}} \\
\left(\mathrm{kJJmol}^{-1}\right) \\
\text { en excès d'O }_{2}\end{array}$ \\
\hline Liaisons C-H & 153 & 97 \\
Liaisons O-H (alcools) & 171 & 89 \\
\hline
\end{tabular}

PEI exposés dans l'air entre 200 et $250^{\circ} \mathrm{C}$. À 220 et $250^{\circ} \mathrm{C}$, on distingue clairement deux comportements sucessifs: tout d'abord, une diminution initiale de $\mathrm{T}_{\mathrm{g}}$ qui traduit une prédominance relative des coupures de chaîne sur la réticulation dès le début de l'exposition; ensuite, une forte augmentation de $\mathrm{T}_{\mathrm{g}}$ au-dessus de sa valeur initiale, indiquant que la réticulation devient à son tour prédominante. Ces deux phénomènes (coupures de chaîne et réticulation) sont fortement thermo-activés. À $200^{\circ} \mathrm{C}$, on soupçonne que ce scénario est toujours valide, mais qu'il se produira après une (longue) période d'induction de l'ordre de $5000 \mathrm{~h}$.

Des analyses DSC ont également été réalisées sur des films de PEI après vieillissement sous différentes pressions partielles d'oxygène comprises entre 0,21 et 10 bars à $220^{\circ} \mathrm{C}$. Les évolutions de $\left(\mathrm{T}_{\mathrm{g}}-\mathrm{T}_{\mathrm{g} 0}\right)$ et de $(\mathrm{X}-\mathrm{S})$ sont données sur la figure 10. On retrouve les mêmes comportements que dans l'air. Cependant, la chûte de $\mathrm{T}_{\mathrm{g}}$ s'accentue avec l'augmentation de la pression partielle d'oxygène. D'une manière générale, la pression partielle d'oxygène accélère les cinétiques de coupure de chaîne et de réticulation jusqu'à une pression critique de l'ordre de 0,6 bar à $220^{\circ} \mathrm{C}$, qui a déjà été déterminée, mais avec une plus grande précision, par spectroscopie IRTF. À noter qu'il a été difficile de suivre la réticulation au-dessus de la valeur initiale de $T_{g}$, notamment sous forte pression partielle d'oxygène, en raison de l'extrême fragilité des films de PEI.

Les valeurs du temps d'induction des modifications macromoléculaires $\left(t_{i}\right)$ et des vitesses maximales de coupure de chaîne $\left(\mathrm{r}_{\mathrm{s}}\right)$ et de réticulation $\left(\mathrm{r}_{\mathrm{x}}\right)$ déterminées à $220^{\circ} \mathrm{C}$ ont été portées en fonction de la pression partielle d'oxygène sur la figure 11 . On constate que les valeurs de $r_{s}$ et $r_{x}$ sont comparables. De plus, les courbes présentent une allure hyperbolique et atteignent un plateau caractéristique du régime en excès d'oxygène à partir de $\mathrm{P}_{\mathrm{c}}=3$ bars. Les valeurs de $t_{i}, r_{s}$ et $r_{x}$ en excès d'oxygène à $220^{\circ} \mathrm{C}$ sont récapitulées dans le tableau 4 où elles sont comparées aux valeurs obtenues pour les produits de dégradation.

On remarque que les coupures de chaîne sont détectées avant la disparition des groupes $\mathrm{CH}_{3}$ et la formation des alcools, quelle que soit la pression partielle d'oxygène. On peut donc en déduire qu'elles se produisent majoritairement au niveau des liaisons $\mathrm{Ph}-\mathrm{C}\left(\mathrm{CH}_{3}\right)_{2}$ dans les étapes d'amorçage par thermolyse, mais aussi par décomposition unimoléculaire des hydroperoxydes (au cours du réarrangement par coupure $\beta$ des radicaux alkoxyles $\mathrm{PO} \mathrm{O}^{\bullet}$ ). Cette dernière réaction est en compétition avec l'arrachement d'hydrogène conduisant à la formation d'alcools (Fig. 8).

Concernant la réticulation, elle devient prédominante sur les coupures de chaîne après une certaine durée d'exposition ( $800 \mathrm{~h}$ à $220^{\circ} \mathrm{C}$ dans l'air), c'est-à-dire lorsque suffisamment de radicaux alkyles $\mathrm{P}^{\bullet}$ et peroxyles $\mathrm{PO}_{2}{ }^{\bullet}$ ont été formés. Elle provient donc du couplage de ces radicaux, comme déjà proposé dans la littérature pour le PC [3]. Les figures 12 et 13 illustrent quelques mécanismes de réticulation possibles pour le PEI. Ils conduisent tous à des ponts $\mathrm{C}-\mathrm{C}$ entre deux chaînes macromoléculaires. En absence d'oxygène, la réticulation résulte directement du 


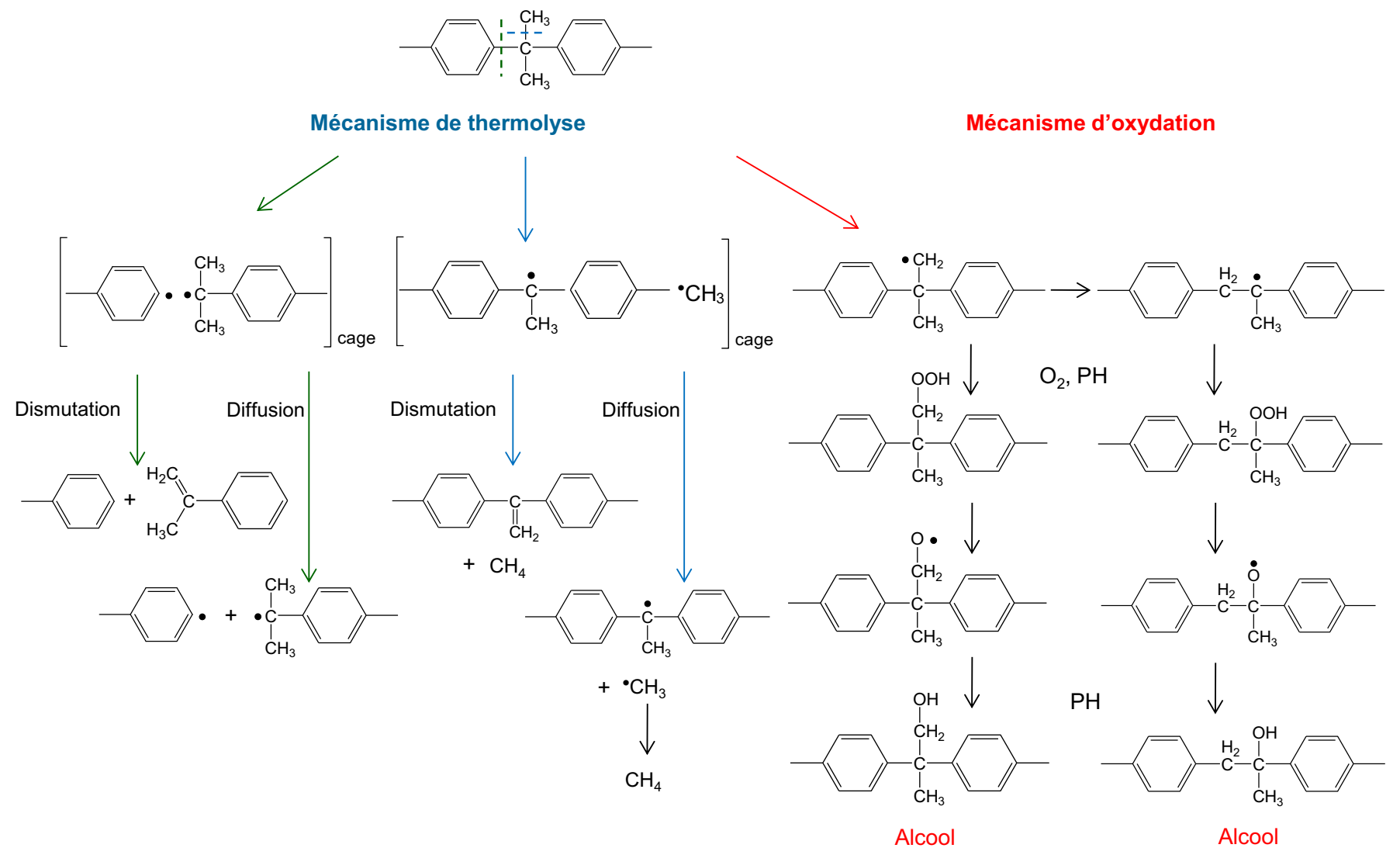

Fig. 8. Mécanisme de dégradation thermique du PEI amorcée par deux voies compétitives $[1,4,7,9]$ : la rupture des liaisons C-C de l'unité isopropylidène (à gauche) et la décomposition unimoléculaire des hydroperoxydes (à droite).

Fig 8. Thermal degradation mechanism of PEI initiated by two competitive paths [1,4,7,9]: the breakdown of C-C bonds of the isopropylidene unit (left) and the unimolecular decomposition of hydroperoxides (right).
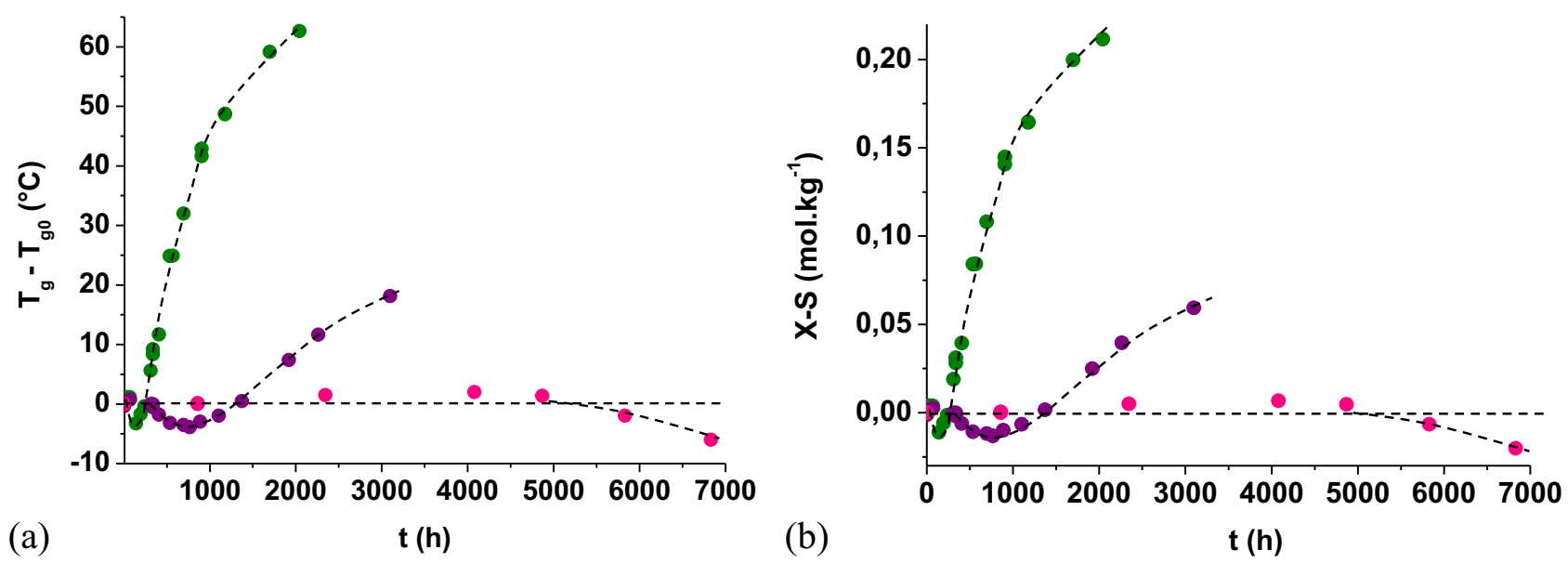

Fig. 9. Évolution des écarts entre la température de transition vitreuse et sa valeur initiale $\left(\mathrm{T}_{\mathrm{g}}-\mathrm{T}_{\mathrm{g} 0}\right)$ (a) et entre les nombres d'actes de réticulation et de coupure de chaîne (X-S) (b) au cours du vieillissement thermique du PEI dans l'air à 200 (en rose), 220 (violet) et $250^{\circ} \mathrm{C}$ (vert).

Fig 9. Changes in the gaps between the glass transition temperature and its initial value $\left(T_{g}-T_{g 0}\right)$ (a) and between the numbers of crosslinking and chain scission events $(X-S)$ (b) during the thermal ageing of PEI in air at 200 (in pink), 220 (violet) and $250^{\circ} \mathrm{C}$ (green). 

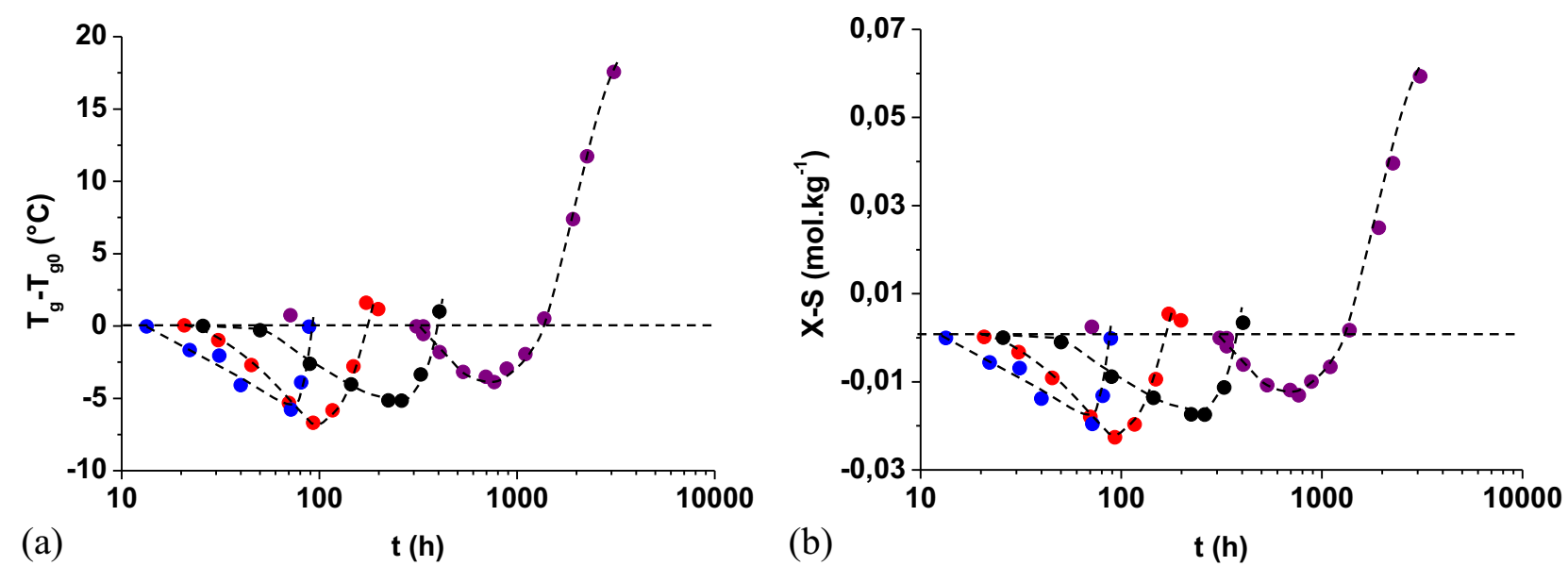

Fig. 10. Évolution des écarts entre la température de transition vitreuse et sa valeur initiale $\left(\mathrm{T}_{\mathrm{g}}-\mathrm{T}_{\mathrm{g} 0}\right)$ (a) et entre les nombres d'actes de réticulation et de coupure de chaîne $(\mathrm{X}-\mathrm{S})(\mathrm{b})$ au cours du vieillissement thermique du PEI sous une pression de 0,21 bar (en violet), 0,4 bar (noir), 3 bars (rouge) et 10 bars d'oxygène (bleu) à $220^{\circ} \mathrm{C}$.

Fig 10. Changes in the gaps between the glass transition temperature and its initial value $\left(T_{g}-T_{g 0}\right)$ (a) and between the numbers of crosslinking and chain scission events $(X-S)$ (b) during the thermal ageing of PEI under an oxygen pressure of 0.21 bar (in violet), 0.4 bar (black), 3 bars (red) and 10 bars (blue) at $220^{\circ} \mathrm{C}$.
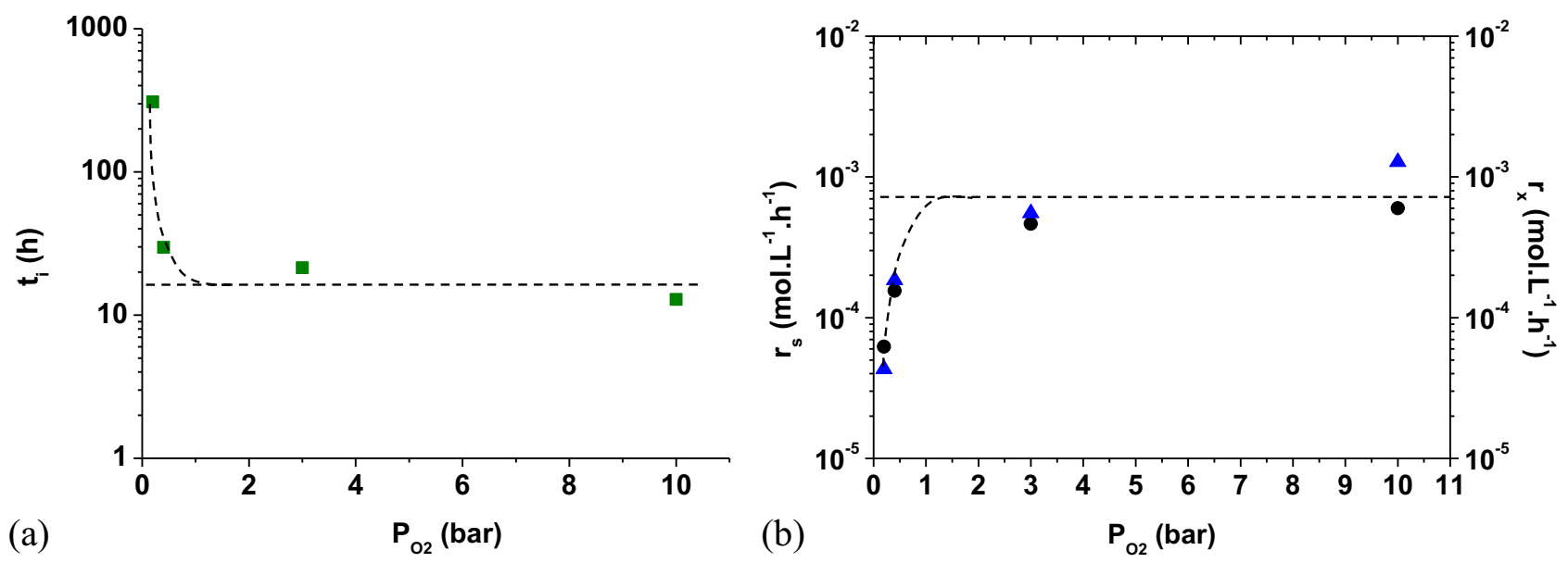

Fig. 11. Variation du temps d'induction des modifications macromoléculaires et des vitesses maximales de coupure de chaîne ( $)$ et de réticulation $(\boldsymbol{\Delta})$ avec la pression partielle d'oxygène à $220^{\circ} \mathrm{C}$.

Fig 11. Changes in the induction time of macromolecular modifications and the maximum rates of chain scission

and crosslinking (A) with oxygen partial pressure at $220^{\circ} \mathrm{C}$.

Tableau 4. Pression critique $\left(\mathrm{P}_{\mathrm{c}}\right)$, temps d'induction $\left(\mathrm{t}_{\mathrm{i}}\right)$ et vitesse maximale $(\mathrm{r})$ des modifications moléculaires et macromoléculaires en excès d'oxygène à $220^{\circ} \mathrm{C}$.

Table 4. Critical pressure $\left(P_{c}\right)$, induction time $\left(t_{i}\right)$ and maximum rate $(r)$ for molar and macromolecular modifications in oxygen excess at $220^{\circ} \mathrm{C}$.

\begin{tabular}{llll}
\hline & $\begin{array}{l}\mathrm{P}_{\mathrm{c}} \\
(\text { bar })\end{array}$ & $\begin{array}{l}\mathrm{t}_{\mathrm{i}} \text { en } \\
\text { excès } \\
\mathrm{d}^{\prime} \mathrm{O}_{2}(\mathrm{~h})\end{array}$ & $\begin{array}{l}\mathrm{r} \text { en } \\
\text { excès d' } \mathrm{O}_{2} \\
\left(\mathrm{~mol}^{-1} \mathrm{~L}^{-1} \cdot \mathrm{h}^{-1}\right)\end{array}$ \\
\hline Produits de dégradation & 0,6 & 53 & $4,3.10^{-3}$ \\
Coupures de chaîne & 3 & 17 & $5,3.10^{-4}$ \\
Réticulation & 3 & - & $9,2.10^{-4}$ \\
\hline
\end{tabular}

couplage des radicaux primaires $\mathrm{P}^{\bullet}$ (Fig. 12). En défaut d'oxygène, comme les ponts peroxydes POOP ne peuvent pas survivre aux températures étudiées (typiquement lorsque $\mathrm{T} \geq 180^{\circ} \mathrm{C}$ ), ils se décomposent presque instantanément pour former deux radicaux $\mathrm{PO}^{\bullet}$ qui peuvent ensuite se réarranger de diverses façons et, en particulier, en radicaux tertiaires $\mathrm{P}^{\bullet}$ par coupure $\beta$ (Fig. 13). Ces derniers peuvent ensuite se coupler pour former un nœud de réticulation. Le même schéma réactionnel peut être proposé en excès d'oxygène, juste après la rapide décomposition des ponts tétraoxydes POOOOP qui sont très instables (Fig. 14).

Enfin, on remarque que les modifications macromoléculaires sont beaucoup plus lentes que la disparition des groupes $\mathrm{CH}_{3}$ ou la formation des alcools (Tab. 4). 


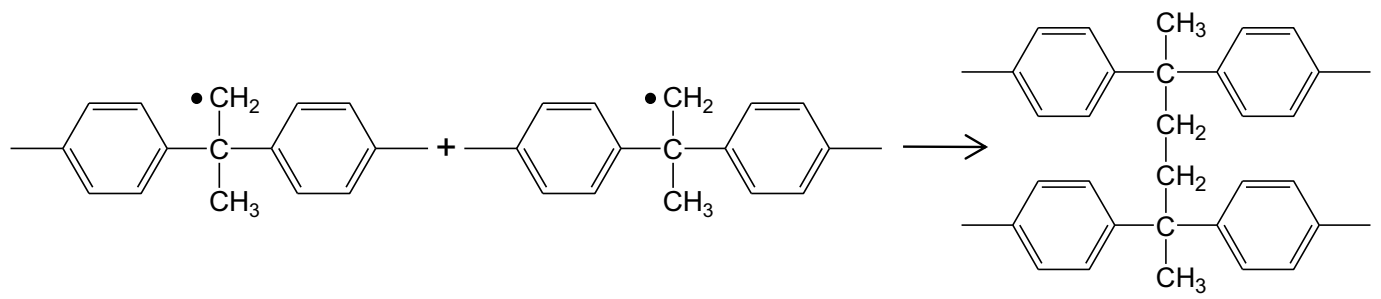

Fig. 12. Réaction de terminaison par couplage des radicaux $\mathrm{P}^{\bullet}$ entraînant la formation d'un pont $\mathrm{C}-\mathrm{C}$ entre deux chaînes macromoléculaires et ainsi, d'un nœud de réticulation.

Fig 12. Termination by coupling of $P^{\bullet}$ radicals leading to the formation of a $C-C$ bridge between two macromolecular chains and thus, to a crosslinking node.
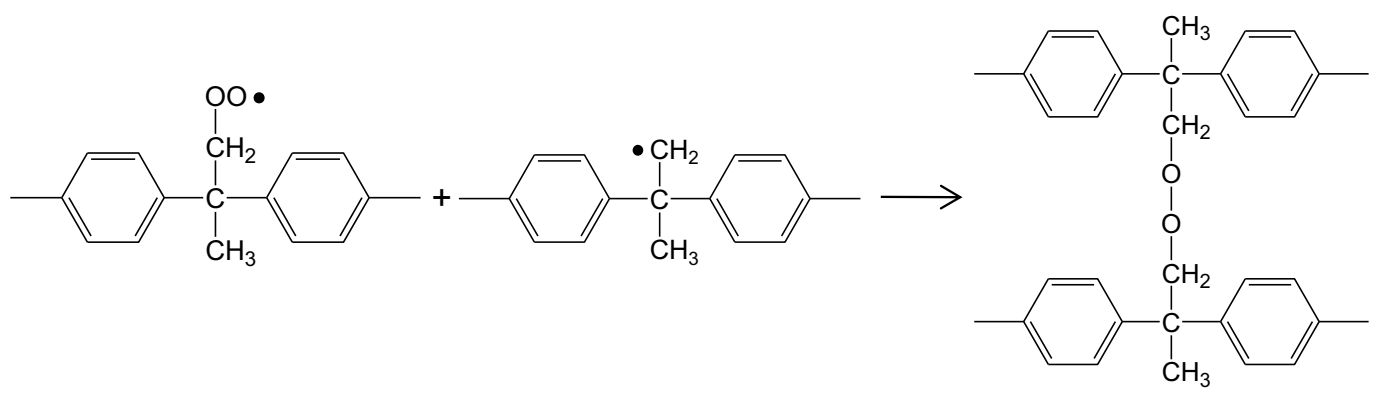

Fig. 13. Réaction de terminaison par couplage des radicaux $\mathrm{P}^{\bullet}$ et $\mathrm{PO}_{2} \bullet$ entraînant la formation d'un pont $\mathrm{C}-\mathrm{C}$ entre deux chaînes macromoléculaires et ainsi, d'un nœud de réticulation.

Fig 13. Termination by coupling of $P^{\bullet}$ et $\mathrm{PO}_{2}^{\bullet}$ radicals leading to the formation of a $\mathrm{C}-\mathrm{C}$ bridge between two macromolecular chains and thus, to a crosslinking node.
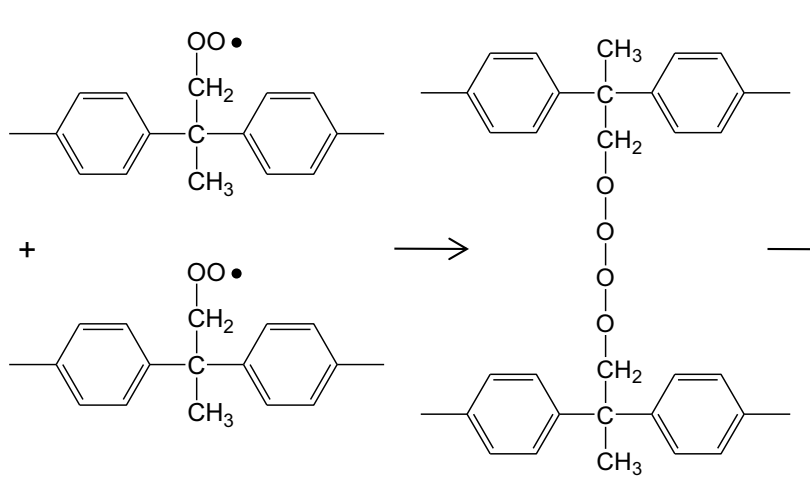
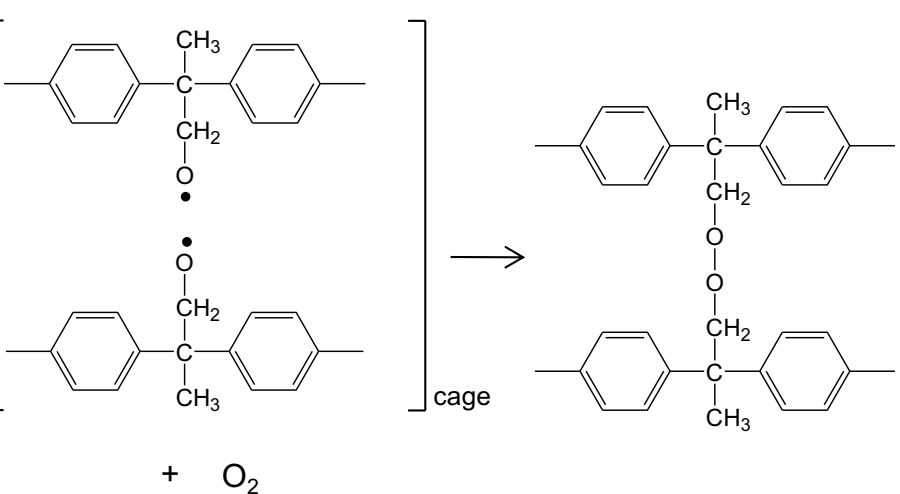

Fig. 14. Réaction de terminaison par couplage des radicaux $\mathrm{PO}_{2}$ entraînant la formation d'un pont C-C entre deux chaînes macromoléculaires et ainsi, d'un nœud de réticulation.

Fig 14. Termination by coupling of $\mathrm{PO}_{2}{ }^{\bullet}$ radicals leading to the formation of a $C-C$ bridge between two macromolecular chains and thus, to a crosslinking node.

\subsection{Conséquences de l'oxydation sur les propriétés élastiques}

Les profils de module élastique générés par l'oxydation dans l'épaisseur des plaquettes de $3 \mathrm{~mm}$ d'épaisseur de PEI ont été déterminés par micro-indentation. À titre d'exemple, les profils obtenus avant et après $1966 \mathrm{~h}$ d'exposition dans l'air à $250^{\circ} \mathrm{C}$ sont rapportés sur la figure 15 . Comme attendu, le profil initial est quasiment plat. La valeur moyenne correspondante $\mathrm{E}_{\mathrm{Echant}} \mathrm{du}$ module élastique est de 5,0 $\pm 0,2 \mathrm{GPa}$. Elle est légèrement inférieure à la valeur du module d'Young déterminée par traction uniaxiale sur des éprouvettes de type haltère : $3,5 \pm 0,1 \mathrm{GPa}$. Pour cette raison, dans cette étude, un facteur correctif de 0,7 a été systématiquement appliqué au module d'indentation pour remonter à la valeur du module d'Young.

$\mathrm{Au}$ cours du vieillissement thermique, on observe une augmentation du module d'Young dans la couche superficielle oxydée (Fig. 15). Cette augmentation se corrèle parfaitement avec la disparition des groupes $\mathrm{CH}_{3}$ déterminée par spectroscopie IRTF en mode ATR (Fig. 16). De plus, les épaisseurs de couche oxydée déterminées à l'aide 


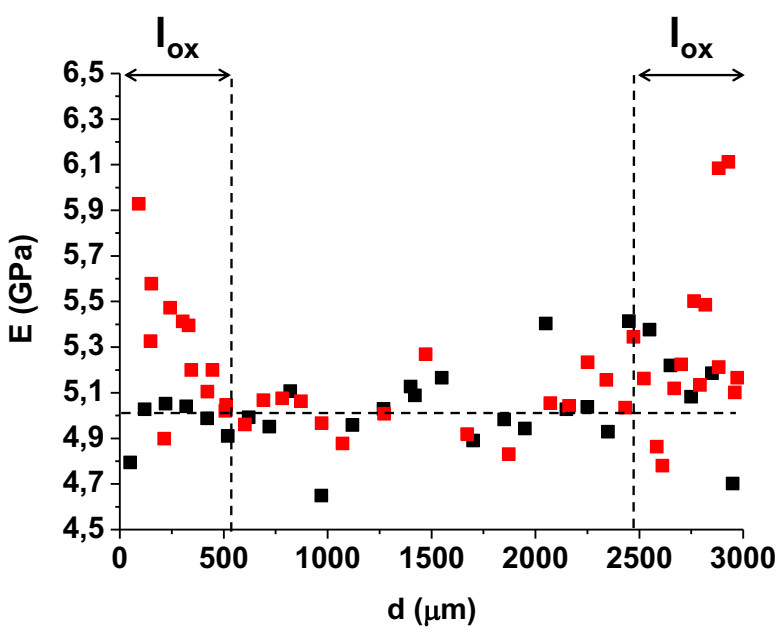

Fig. 15. Profil de module d'Young dans l'épaisseur d'une palquette de PEI avant (en noir) et après (rouge) $1966 \mathrm{~h}$ d'exposition dans l'air à $250^{\circ} \mathrm{C}$.

Fig 15. Profile of the Young's modulus throughout the thickness of the PEI plate before (in black) and after (red) 1966 h of exposure in air at $250^{\circ} \mathrm{C}$.

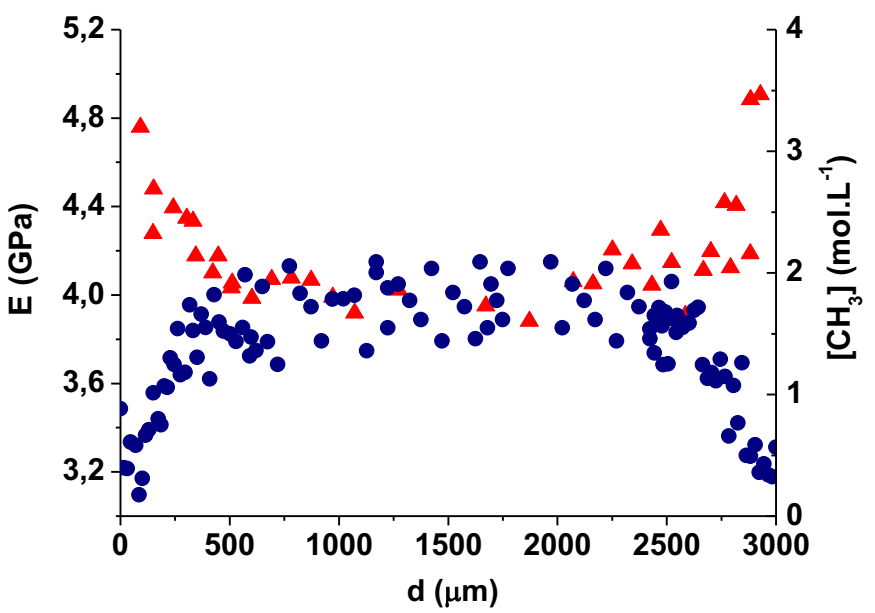

Fig. 16. Comparaison des profils de dégradation déterminés dans l'épaisseur d'une plaquette de PEI par spectroscopie IRTF (๑) et par micro-indentation ( $\mathbf{A})$ après $1966 \mathrm{~h}$ d'exposition dans l'air à $250^{\circ} \mathrm{C}$.

Fig 16. Comparison of the degradation profiles determined throughout the thickness of a PEI plate by FTIR spectrophotometry ( $\mathbf{O})$ and micro-indentation ( $\mathbf{\Delta}$ ) after 1966h of exposure in air at $250^{\circ} \mathrm{C}$.

de ces deux techniques (micro-indentation et spectroscopie IRTF) sont comparables. Elles sont du même ordre de grandeur que les épaisseurs de couche oxydée déterminées à l'aide d'une troisième technique : la microscopie optique. À titre d'exemple, la figure 17 présente des micrographies obtenues après quatre durées d'exposition dans l'air à $220^{\circ} \mathrm{C}$. On voit clairement que l'oxydation s'accompagne d'un changement de couleur (brunissement) dans les couches superficielles des échantillons.
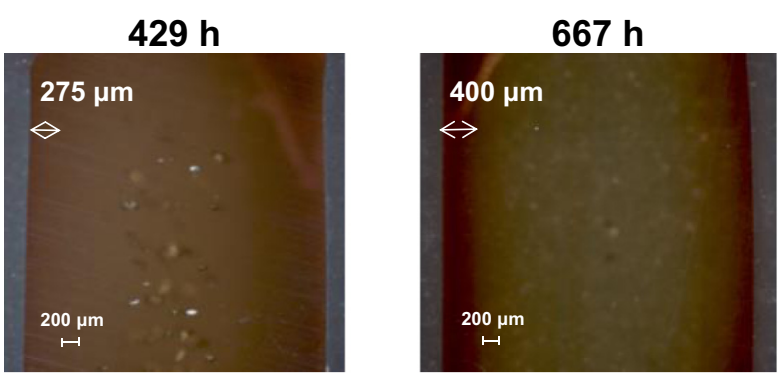

$1270 \mathrm{~h}$
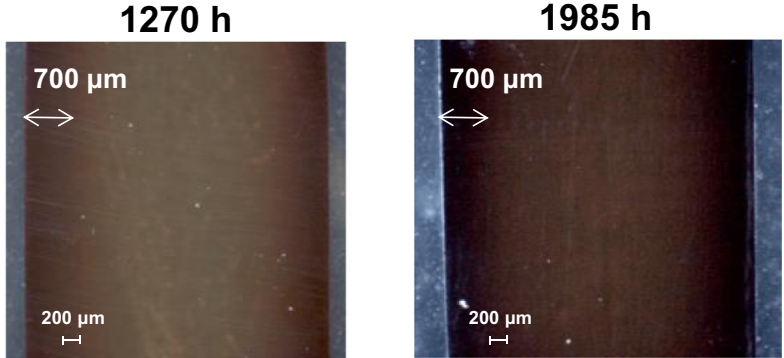

Fig. 17. Observation au microscope optique (avec un grosissement $\times 2,5)$ de la couche superficielle oxydée du PEI après différentes durées d'exposition dans l'air à $220^{\circ} \mathrm{C}$.

Fig 17. Examination by optical microscopy (with a magnification of $\times 2,5)$ of the superficial oxidized layer of PEI after different durations of exposure in air at $220^{\circ} \mathrm{C}$.

Tableau 5. Épaisseur moyenne de couche oxydée du PEI déterminée par micro-indentation, spectroscopie IRTF ou microscopie optique après différentes durées d'exposition dans l'air à 220 et $250^{\circ} \mathrm{C}$.

Table 5. Average thickness of oxidized layer of PEI determined by micro-indentation, FTIR spectrophotometry or optical microscopy after different durations of exposure in air at 220 and $250^{\circ} \mathrm{C}$.

\begin{tabular}{lrl}
\hline $\mathrm{T}\left({ }^{\circ} \mathrm{C}\right)$ & $\mathrm{t}(\mathrm{h})$ & $\mathrm{l}_{\text {ox }}(\mu \mathrm{m})$ \\
\hline 220 & 429 & $275 \pm 25$ \\
& 667 & $400 \pm 50$ \\
& 1270 & $750 \pm 50$ \\
& 1985 & $750 \pm 50$ \\
250 & 94 & $225 \pm 25$ \\
& 670 & $350 \pm 75$ \\
& 1273 & $490 \pm 60$ \\
& 1966 & $550 \pm 50$ \\
\hline
\end{tabular}

Les épaisseurs moyennes de couche oxydée déterminées dans l'air à 220 et $250^{\circ} \mathrm{C}$ à l'aide de ces trois techniques sont récapitulées dans le tableau 5 .

Cependant, l'augmentation du module d'Young n'est pas la conséquence directe de l'oxydation, mais d'un vieillissement physique révélé par l'apparition et la croissance d'un endotherme de relaxation enthapique (ou structurale) dans la zone de transition vitreuse sur les thermogrammes DSC (Fig. 18). 
Ainsi, si le PEI est initialement à l'état vitreux (cas du vieillissement à 180 et $200{ }^{\circ} \mathrm{C}$ ), l'augmentation du module d'Young est détectée dès le début de l'exposition. En revanche, si le PEI est initialement à l'état caoutchoutique (cas du vieillissement à 220 et $250^{\circ} \mathrm{C}$ ), l'augmentation du module d'Young n'est détectée que lorsque la $T_{g}$ devient supérieure à la température d'exposition, typiquement après $1500 \mathrm{~h}$ dans l'air à $220^{\circ} \mathrm{C}$ et après $600 \mathrm{~h}$ dans l'air à $250^{\circ} \mathrm{C}$.

La figure 19 rapporte l'évolution de l'enthalpie et de la température du pic endothermique au cours du vieillissement thermique du PEI dans l'air entre 180 et $250{ }^{\circ} \mathrm{C}$. Ces deux grandeurs augmentent avec le temps d'exposition jusqu'à atteindre un état d'équilibre thermodynamique à 180 et $250^{\circ} \mathrm{C}$. Cependant, à $200^{\circ} \mathrm{C}$, elles diminuent à plus long terme.

L'évolution de l'enthalpie du pic endothermique est liée à l'évolution de $\mathrm{T}_{\mathrm{g}}$, c'est-à-dire aux modifications macro-

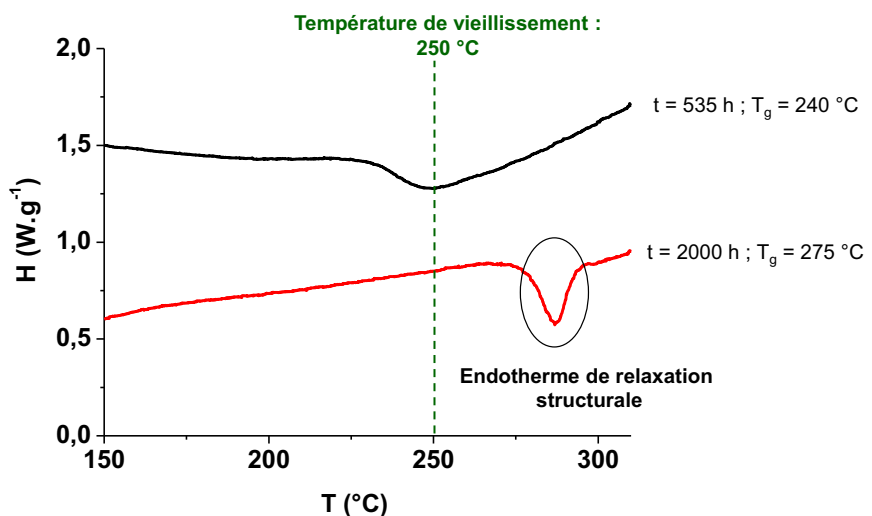

Fig. 18. Thermogrammes DSC de films de PEI après $535 \mathrm{~h}$ et $2000 \mathrm{~h}$ d'exposition dans l'air à $250^{\circ} \mathrm{C}$.

Fig 18. DSC thermogrammes of PEI films after $535 \mathrm{~h}$ and $2000 \mathrm{~h}$ of exposure in air at $250^{\circ} \mathrm{C}$.

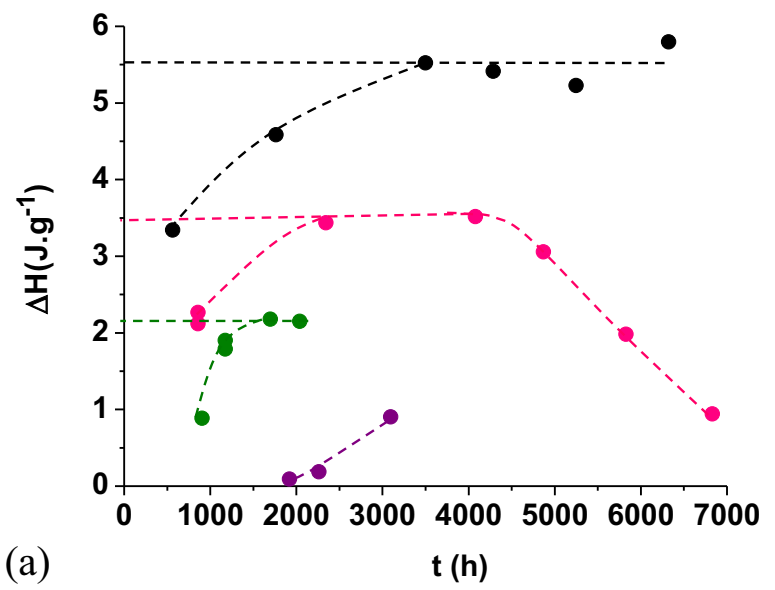

moléculaires. En effet, les coupures de chaine favorisent la mobilité moléculaire, en l'occurrence le changement de conformation des chaines, car elles détruisent progressivment le réseau d'enchevêtements. Les coupures de chaine sont prédominantes en début d'exposition, mais la formation de structures macromoléculaires de plus en plus ramifiées (par couplage des radicaux $\mathrm{P}^{\bullet}$ et $\mathrm{PO}_{2}^{\bullet}$ ) réduit aussi progressivement la capacité du matériau à subir une relaxation structurale.

Sur cette base, une corrélation a pu être établie entre les valeurs locales du module d'Young, déterminées par microindentation avant et après exposition des plaquettes de PEI dans l'air à 220 et $250^{\circ} \mathrm{C}$, et les valeurs correspondantes de l'enthalpie de relaxation déterminées par DSC (Fig. 20).

Elle s'écrit:

$$
E=5,2+0,35 \times \Delta H .
$$

Les valeurs globales du module d'Young $\mathrm{E}_{\mathrm{CO}}$ de la couche oxydée des plaquettes de PEI vieillies dans l'air à 220 et $250^{\circ} \mathrm{C}$, ont été calculées à l'aide de l'équation 4 . Ces valeurs ont été portées en fonction de l'inverse de la température de transition vitreuse sur la figure 21. On remarque que tous les points se placent autour d'une droite maîtresse unique dont l'équation vérifie la relation de Gilbert et al. [24] :

$$
E_{c o}(T)=E(0 K)\left(1-\alpha_{E} \frac{T}{T_{g}}\right),
$$

où $\mathrm{E}(0 \mathrm{~K})$ est la valeur du module d'Young à $0 \mathrm{~K}$ et $\alpha_{\mathrm{E}}$ est une constante.

Ainsi, pour le PEI, on trouve: $\mathrm{E}(0 \mathrm{~K})=4,1 \mathrm{GPa}$ et $\alpha_{E}=0,18$. Notons que cette dernière valeur est du même ordre de grandeur que la valeur déjà proposée dans la littérature par Gilbert et al. [24] $\left(\alpha_{E}=0,3\right)$ qui serait, d'après ces auteurs, quasi-indépendante de la structure du polymère.

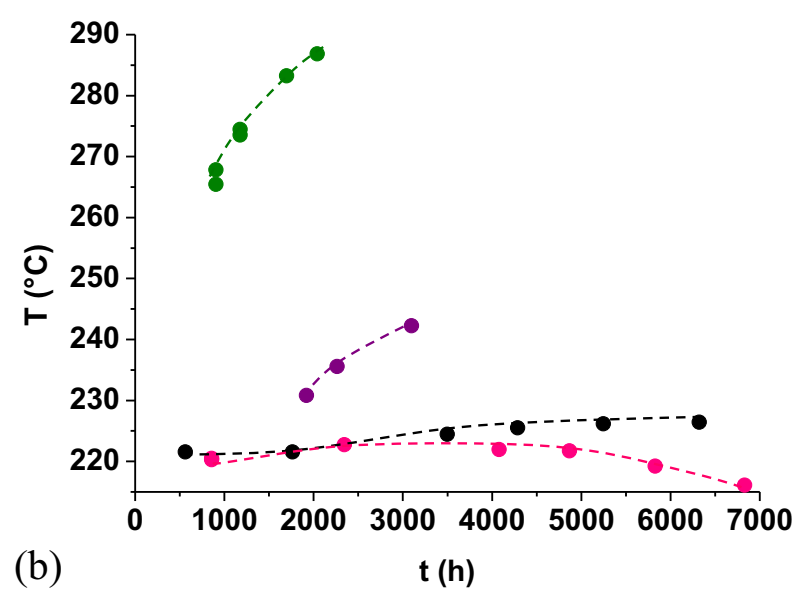

Fig. 19. Évolution de l'enthalpie (a) et de la température du pic endothermique (b) au cours du vieillissement thermique du PEI dans l'air à 180 (noir), 200 (rose), 220 (violet) et $250^{\circ} \mathrm{C}$ (vert).

Fig 19. Changes in the enthalpy (a) and the temperature of the endothermic peak (b) during the thermal ageing of PEI in air at 180 (black), 200 (pink), 220 (violet) and $250^{\circ} \mathrm{C}$ (green). 


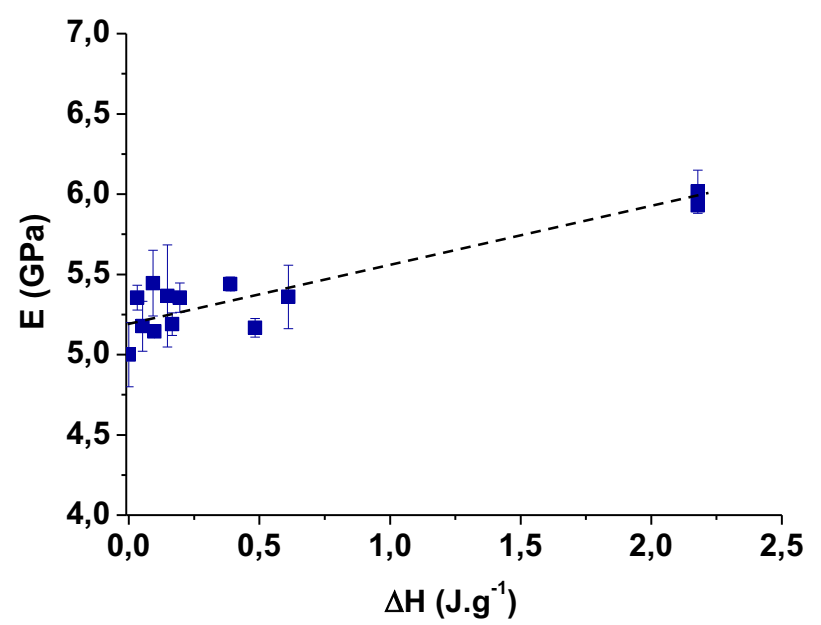

Fig. 20. Variation locale du module d'Young avec l'enthalpie de relaxation au cours du vieillissement thermique du PEI dans l'air à 220 et $250^{\circ} \mathrm{C}$.

Fig 20. Local changes in the Young's modulus with the relaxation enthalpy during the thermal ageing of PEI in air at 220 and $250^{\circ} \mathrm{C}$.

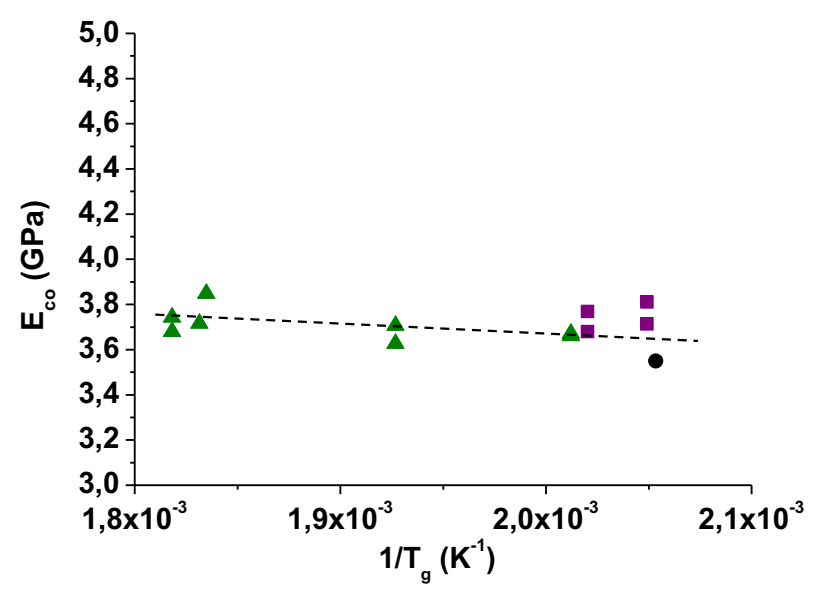

Fig. 21. Variation du module d'Young de la couche oxydée des plaquettes de PEI en fonction de l'inverse de sa $\mathrm{T}_{\mathrm{g}}$ avant $(\mathbf{O})$ et après exposition dans l'air à $220(\square)$ et $250^{\circ} \mathrm{C}(\boldsymbol{\Delta})$.

Fig 21. Changes in the Young's modulus of the oxidized layer of PEI plates in fonction of its reciprocal $T_{q}$ before (O) and after exposure in air at 220 (口) and $250^{\circ} \mathrm{C}(\mathbf{\Delta})$.

\section{Conclusion}

La dégradation thermique de la matrice PEI a été étudiée à l'état caoutchoutique $\left(220\right.$ et $\left.250^{\circ} \mathrm{C}\right)$ et à l'état vitreux ( 180 et $200^{\circ} \mathrm{C}$ ) sous des pressions partielles d'oxygène comprises entre 0,21 et 50 bars, c'est-à-dire dans des domaines de température et de pression partielle d'oxygène encore totalement inexplorés. L'analyse à l'échelle moléculaire, par spectroscopie IRTF, a permis de confirmer les mécanismes de thermolyse et de thermo-oxydation proposés dans la littérature impliquant les groupes $\mathrm{CH}_{3}$ de l'unité isopropylidène du motif bisphénol $\mathrm{A}$, mais aussi d'identifier les alcools comme principaux produits d'oxy- dation. L'analyse aux échelles supérieures, par DSC puis par micro-indentation, a permis de montrer d'une part, que les coupures de chaînes sont majoritaires en début d'exposition et que la réticulation devient prédominante à plus long terme, quelle que soit la pression partielle d'oxygène. D'autre part, le contrôle de l'oxydation par la diffusion se traduit par la formation d'une couche superficielle oxydée dans laquelle les propriétés élastiques $\mathrm{du}$ PEI sont significativement impactées. Cependant, l'augmentation du module d'Young n'est pas une conséquence directe de l'oxydation, mais plutôt d'un vieillissement physique.

Dans une prochaine communication, un modèle cinétique sera dérivé du schéma mécanistique d'oxydation du PEI pour être interfacé avec les relations structure/ propriété identifiées dans cette étude (en particulier, avec les équations 7 et 9 ). Cet outil théorique permettra de prédire non seulement l'état de dégradation du PEI, mais aussi l'évolution de ses propriétés thermomécaniques au cours du vieillissement thermique. La validité de cet outil sera vérifiée à partir de l'ensemble des données expérimentales récoltées dans cette étude.

\section{References}

1. S. Carroccio, C. Puglisi, G. Montaudo, New vistas in polymer degradation. thermal oxidation processes in Poly(ether imide), Macromolecules 38, 6849 (2005)

2. A. Rivaton, Recent advances in bisphenol-A polycarbonate photodegradation, Polym. Degrad. Stab. 49, 163 (1995)

3. S. Collin, P.O. Bussière, S. Thérias, J.M. Lambert, J. Perdereau, J.L. Gardette, Physicochemical and mechanical impacts of photo-ageing on bisphenol a polycarbonate, Polym. Degrad. Stab. 97, 2284 (2012)

4. A. Rivaton, B. Mailhot, J. Soulestin, H. Varghese, J.L. Gardette, Comparison of the photochemical and thermal degradation of bisphenol-A polycarbonate and trimethylcyclohexane-polycarbonate, Polym. Degrad. Stab. 75, 17 (2002)

5. S. Kuroda, I. Mita, Degradation of aromatic polymers-II. The crosslinking during thermal and thermo-oxidative degradation of a polyimide, Eur. Polym. J. 25, 611 (1989)

6. M.A. Corres, M. Zubitur, M. Cortazar, A. Múgica, Thermal and thermo-oxidative degradation of poly(hydroxy ether of bisphenol-A) studied by TGA/FTIR and TGA/MS, J. Anal. Appl. Pyrolysis 92, 407 (2011)

7. S. Carroccio, C. Puglisi, G. Montaudo, Thermal degradation mechanisms of polyetherimide investigated by direct pyrolysis mass spectrometry, Macromol. Chem. Phys. 200, 2345 (1999)

8. G. Lisa, C. Hamciuc, E. Hamciuc, N. Tudorachi, Thermal and thermo-oxidative stability and probable degradation mechanism of some polyetherimides, J. Anal. Appl. Pyrolysis 118, 144 (2016)

9. H. Farong, W. Xueqiu, L. Shijin, The thermal stability of polyetherimide, Polym. Degrad. Stab. 18, 247 (1987)

10. R.A. Dine-Hart, D.B.V. Parker, W.W. Wright, Oxidative degradation of a polyimide film: I. Initial studies, Br. Polym. J. 3, 222 (1971)

11. L. Augh, J.W. Gillespie, B.K. Fink, Degradation of continuous carbon fiber reinforced polyetherimide composites during induction heating, J. Thermoplast. Compos. Mater. 14, 96 (2001) 
12. S. Commereuc, D. Vaillant, J.L. Philippart, J. Lacoste, J. Lemaire, D.J. Carlsson, Photo and thermal decomposition of iPP hydroperoxides, Polym. Degrad. Stab. 57, 175 (1997)

13. D.J. Carlsson, R. Brousseau, D.M. Wiles, Reactions of sulfur dioxide with oxidized polyolefins, Polym. Degrad. Stab. 15, 67 (1986)

14. V.A. Matyshak, O.V. Krylov, Problems of quantitative spectroscopic measurements in heterogeneous catalysis: molar absorption coefficients of vibrations in adsorbed substances, Kinet. Catal. 43, 391 (2002)

15. T. Iqbal, B.J. Briscoe, P.F. Luckham, Surface plasticization of Poly(ether ether ketone), Eur. Polym. J. 47, 2244 (2011)

16. W.C. Oliver, G.M. Pharr, An improved technique for determining hardness and elastic modulus using load and displacement sensing indentation experiments, J. Mater. Res. 7, 1564 (1992)

17. P. Clément, Détermination des propriétés mécaniques de céramiques poreuses par essais de microindentation instrumentée sphérique, Thèse de doctorat, INSA Lyon, 2013
18. J.G. Calvert, J.N. Pitts, Photochemistry, Wiley, New York, 1966

19. X. Colin, L. Audouin, J. Verdu, Determination of thermal oxidation rate constants by an inverse method. Application to polyethylene, Polym. Degrad. Stab. 86, 309 (2004)

20. T.G. Fox, P.J. Flory, Second-order transition temperatures and related properties of polystyrene. I. Influence of molecular weight, J. Appl. Phys. 21, 581 (1950)

21. J. Bicerano, Prediction of polymer properties, 3rd ed, Marcel Dekker, New York, 2002

22. R. Simha, R.F. Boyer, On a general relation involving the glass temperature and coefficients of expansion of polymers, J. Chem. Phys. 37(5), 1003 (1962)

23. E. Richaud, P. Ferreira, L. Audouin, X. Colin, J. Verdu, C. Monchy-Leroy, Radiochemical ageing of poly(ether ether ketone), Eur. Polym. J. 46, 731 (2010)

24. D.G. Gilbert, M.F. Ashby, P.W.R. Beaumont, Modulusmaps for amorphous polymers, J. Mater. Sci., 213194 (2015)

Citation de l'article : Emilie Courvoisier, Yoann Bicaba, Xavier Colin, Analyse de la dégradation thermique du Poly(éther imide), Matériaux \& Techniques 105, 402 (2017) 